\title{
Fully coupled driving safety analysis of moving traffic on long-span bridges subjected to crosswind
}

\author{
Yufen Zhou ${ }^{1}$ and Suren Chen ${ }^{2 *}$
}

\begin{abstract}
High-sided vehicles often experience considerable single-vehicle accident risks under crosswinds. Longspan cable-supported bridges are usually flexible, wind-sensitive and support considerable amount of vehicles on a daily basis. When vehicles are driven through a long-span bridge, the complex dynamic interactions among wind, vehicles and the bridge significantly affect not only the safety of the bridge members, but also passing vehicles. Therefore, realistic modeling of the bridge-traffic system by characterizing these critical coupling effects becomes essential for rationally assessing traffic safety of passing traffic through a long-span bridge. In most existing studies about traffic safety study on bridges, vehicle safety was assessed for only one single vehicle or uniformly distributed vehicles at a constant driving speed. It is known that the traffic flow on a long-span bridge is typically stochastic and vehicle speeds vary following some traffic rules. Based on the stochastic traffic flow simulation, two new analytical frameworks, one using mode superposition and the other using the finite element (FE) formulation, were proposed recently for the bridge-stochastic traffic system. By considering the fullcoupling effects among all the vehicles of the traffic flow, bridge and wind, the dynamic response of each individual vehicle of the stochastic traffic can be accurately obtained for the first time. Built based on the recent advances made by the authors, an integrated dynamic interaction and safety assessment model of the fully-coupled bridge-traffic system is further developed without considering vehicle aerodynamic interference and shielding effects. Traffic safety of vehicles in the stochastic traffic through the prototype long-span cable-stayed bridge is investigated as a demonstration.
\end{abstract}

\footnotetext{
${ }^{1}$ Ph. D. Candidate, Dept. of Civil \& Environmental Engineering, Colorado State University, Fort Collins, CO 80523, Email: yufzhou@rams.colostate.edu.

$2 *$ Corresponding author, P.E., Associate Professor, Dept. of Civil \& Environmental Engineering, Colorado State University, Fort Collins, CO 80523, Email: suren.chen@ colostate.edu, tel: 970-491-7722, fax: 970-491-7727.
} 
Keywords: long-span bridges; stochastic traffic; crosswind; vehicle safety; accident

\section{Introduction}

Highway vehicles may experience single-vehicle accidents under hazardous driving environments, such as strong crosswind, slippery road surface with rain, snow or ice, etc. (USDOT 2005). For traffic through flexible transportation infrastructures, such as bridges, single-vehicle accident risks were found to increase due to the dynamic coupling effects between the vehicle and the supporting structure (Baker et al. 1999; Guo and Xu 2006). Long-span bridges are usually built across straits or major rivers, and therefore

they are more open than most roads with less blocking effects from surrounding environment, exposing vehicles to stronger crosswind. Long-span cable-supported bridges are flexible, susceptible to wind excitations and support considerable amount of vehicles on a daily basis. In addition, vehicles driven on long-span cable-supported bridges may also be temporarily shielded from the crosswind by the bridge tower or other nearby high-sided vehicles, experiencing sharp crosswind gust as well as higher accident risks. When vehicles are driven through a long-span bridge, the complex dynamic interactions among the wind, vehicles and bridge significantly affect not only the performance of the bridge, but also the safety of passing vehicles (Chen and $\mathrm{Wu}$ 2010; Chen and Cai 2004; Guo and Xu 2006). Therefore, a rational traffic safety assessment of passing traffic through a long-span bridge requires appropriate modeling of the critical coupling effects within the bridge-traffic-wind system.

Baker $(1986,1987,1991,1994)$ started series of studies on the safety of road vehicles under sharp crosswind gusts primarily on roadways. Guo and $\mathrm{Xu}$ (2006) conducted vehicle safety analysis of one single vehicle by considering the bridge-vehicle interaction effects subjected to high wind. In the studies by Chen and Cai (2004) and Chen et al. (2009), the authors developed a local vehicle accident assessment model and combined it with the global bridge-vehicle interaction model to consider the vibrating effects from the bridge structure in the windy environment. Chen and Chen (2010) further developed a general local single-vehicle accident model with new transient dynamic equations and accident criteria to 
consider more realistic weather environment and topographical conditions, which can also be used on bridges with some approximations. In all these existing studies about traffic safety on bridges, vehicle safety was assessed with only one single vehicle at a constant driving speed.

In reality, it is known that the traffic flow on a long-span bridge is typically stochastic and vehicle speeds may vary following some realistic traffic rules (Chen and $\mathrm{Wu} 2011$ ). This is especially true when the bridge span is long and the total number of vehicles on the bridge at a time is not small. Apparently, to assume only one vehicle on the bridge with constant speed cannot reflect the realistic situations on most long-span bridges. Among all the technical hurdles preventing researchers from carrying out more realistic traffic safety assessment, the primary one is the difficulty on reasonably modeling the full dynamic interaction effects of the bridge-traffic system subjected to wind. Chen and $\mathrm{Wu}(2011,2010)$ incorporated the stochastic traffic flow simulation into the bridge-traffic interaction analysis, offering a venue to more realistically simulate the dynamic interactions of multiple-vehicle scenarios from stochastic moving traffic on a long-span bridge. However, the approach was based on the equivalentdynamic-wheel-loading (EDWL) concept (Chen and Wu 2010), which focused on the bridge response without being able to provide accurate estimation of the dynamic response of individual vehicles of the simulated stochastic traffic (Zhou and Chen 2014a). As a result, hurdles still remained on assessing vehicles safety on long-span bridges when stochastic traffic is simulated until recently when some advances on modeling techniques were made.

Recently, Zhou and Chen (2014a, b) proposed new mode-based and FE-based dynamic analysis frameworks of bridge-traffic system by considering the full-coupling effects of all the vehicles, the bridge and the wind simultaneously. The dynamic response of each individual vehicle of the stochastic traffic can be accurately obtained for the first time, which provides essential basis for the advanced traffic safety assessment of more realistic traffic flow. Built based on the recent advances by the authors (Zhou and Chen 2014a, b), the present study reports the efforts on developing an integrated dynamic interaction and safety assessment model of the fully coupled bridge-traffic system. Developed within the same simulation 
framework, the dynamic bridge-traffic interaction analysis is conducted to obtain the vehicle response considering road roughness and wind excitations, followed by vehicle safety assessment of different accident types for the stochastic traffic flow in the windy environment. A prototype long-span cablestayed bridge and the simulated stochastic traffic are studied as a demonstration of the proposed approach. Due to the lack of the reliable experimental data, aerodynamic interference and shielding effects on the vehicles and the bridge are not considered in this study.

\section{Mathematical modeling of the vehicles and the bridge}

\subsection{Modeling of the long-span cable-supported bridge}

A long-span cable-stayed bridge is modeled in this study as a three-dimensional finite element model using two types of finite elements. The bridge girder and pylon are modeled with nonlinear spatial beam element based on Timoshenko beam theory. The axial, bending, torsional warping and shear deformation effects are considered at the same time. The stay cables are modeled with catenary cable elements. They are derived based on the exact analytical expression of differential equations for elastic catenary elements. The geometric nonlinear effect of axial forces on the bridge girder and pylons and the cable tension can be taken into account. The effects of flexibility and large deflection in the cables are also considered in establishing the equilibrium equations of the element. Rayleigh damping is assumed to model the structural damping of the bridge, in which the participating factors for the stiffness and mass matrices are obtained from two structural damping ratios associated with two specific modes.

\subsection{Modeling of the road vehicles}

Three types of vehicles are involved in the present study, which are high-sided heavy trucks, light trucks with medium height and light sedan cars. Each type of vehicle is modeled as several rigid bodies and wheel axles connected by series of springs, dampers and pivots. The suspension system and the elastic tires are modeled as springs in the upper and lower positions, respectively. The energy dissipation is 
achieved by modeling upper and lower viscous dampers for the suspension system. The masses of the suspension system and the tires are assumed to be concentrated on the mass blocks at each side of the vehicle and the masses of the springs and dampers are assumed to be zero. Each main rigid body contains four degrees of freedom, including two translational and two rotational ones. The numerical dynamic model of the heavy truck is composed of two main rigid bodies, three wheel axle sets, twenty-four sets of springs and dampers vertically and laterally, as shown in Fig. 1. The displacement vector $d_{v}$ of the heavy truck model involves 19 independent DOFs including 11 in vertical and 8 in lateral directions, as defined in Eq. (1).

$$
d_{v}=\left\{Z_{r 1} \theta_{r 1} \beta_{r 1} Z_{r 2} \beta_{r 2} Z_{a 1 L} Z_{a 1 R} Z_{a 2 L} Z_{a 2 R} Z_{a 3 L} Z_{a 3 R} Y_{r 1} Y_{r 2} Y_{a 1 L} Y_{a 1 R} Y_{a 2 L} Y_{a 2 R} Y_{a 3 L} Y_{a 3 R}\right\}
$$

where $Z_{r i}$ represents the vertical displacement of the $i^{\text {th }}$ rigid body; $\theta_{r i}$ represents the pitching displacement of the $i^{\text {th }}$ rigid body in the $x$ - $z$ plane; $\beta_{r i}$ represents the rotational displacement of the $i^{\text {th }}$ rigid body in the $y-z$ plane; $Z_{a i L(R)}$ represents the vertical displacement of the $i^{\text {th }}$ wheel axle in the left (right) side; $Y_{r i}$ represents the lateral displacement of the $i^{\text {th }}$ rigid body; $Y_{a i L(R)}$ represents the lateral displacement of the $i^{\text {th }}$ wheel axle in the left (right) side. In Fig. 1, the spring stiffness coefficient $K$ and damping coefficient $C$ for the springs and dampers of the vehicle models are labeled according to the axle number, and " $u$ "(upper) or "l" (lower) denotes the position of the spring or damper, " $y$ " (lateral)- or " $z$ " (vertical) denotes the direction of the movement and " $L$ " (left) or " $R$ " (right) denotes the side of the vehicle. 


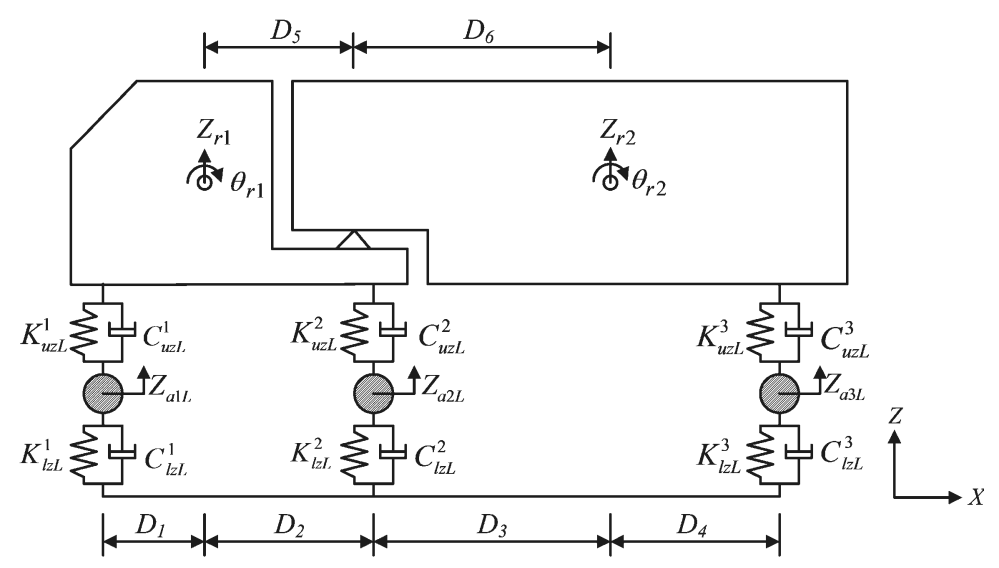

(a) Elevation view

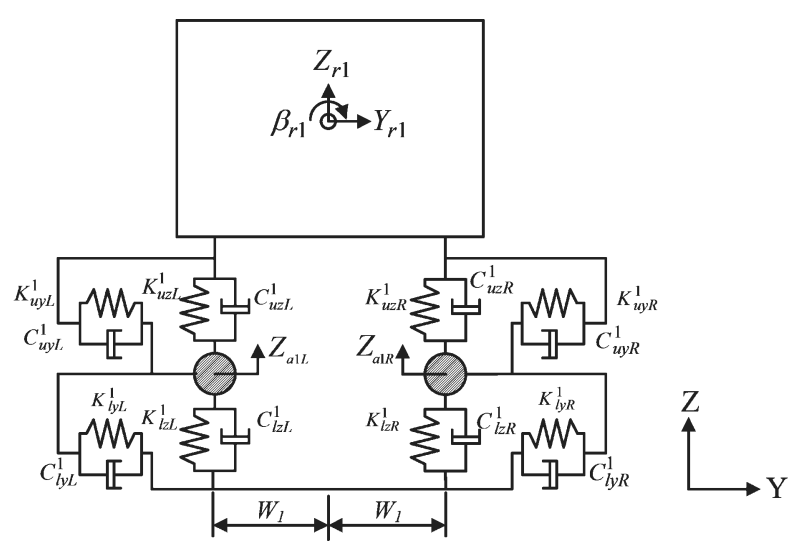

(b) Side view

Fig. 1 The numerical dynamic model for high-sided heavy truck with one trailer

The numerical dynamic model for the light truck and light cars consists of one main rigid body, two wheel axle sets, sixteen sets of springs and dampers. The dynamic models of the light trucks and cars are similar to those of the heavy trucks except with only one rigid body. The elevation view of the light truck and car is shown in Fig. 2 and the side view is similar to that of the heavy truck, which is omitted for brevity purposes. The displacement vector $d_{v}$ for the light truck and car consists of 12 degrees of freedom 
including 7 independent vertical and 5 lateral ones respectively, as expressed in Eq. (2). The variables in the displacement vector are named using the same rules as those of the heavy truck.

$$
d_{v}=\left\{Z_{r 1} \theta_{r 1} \beta_{r 1} Z_{a 1 L} Z_{a 1 R} Z_{a 2 L} Z_{a 2 R} Y_{r 1} Y_{a 1 L} Y_{a 1 R} Y_{a 2 L} Y_{a 2 R}\right\}
$$

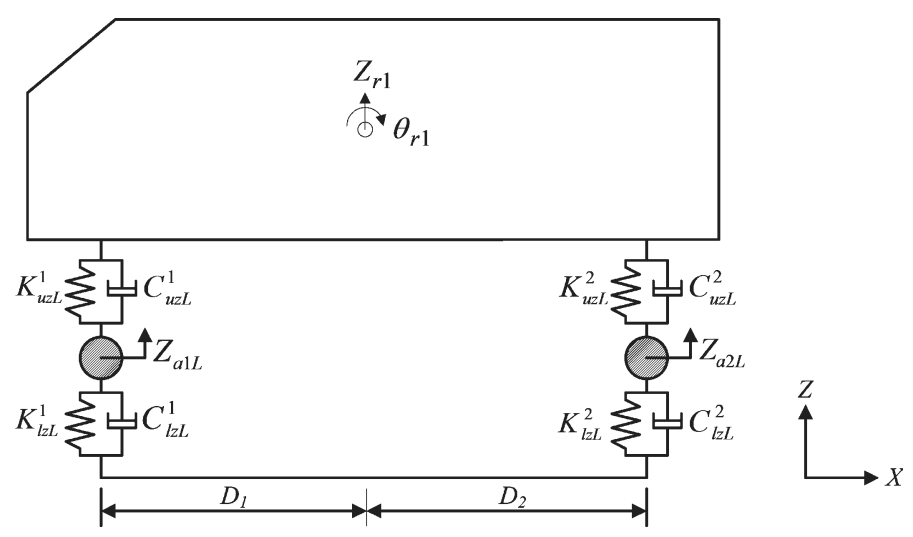

Fig. 2 Elevation view of light truck and light sedan car

\section{Modeling of the dynamic excitations}

\subsection{Modeling of road surface roughness}

The road surface roughness is an important source of the coupling effects for a bridge-traffic system. The roughness on the approaching road and the bridge deck is modeled as a stationary Gaussian random process with zero mean value. The power spectral density function suggested by Huang and Wang (1992) is adopted in the present study, shown in Eq. (3).

$$
S(\bar{\phi})=A_{r}\left(\frac{\bar{\phi}}{\bar{\phi}_{0}}\right)^{-2}
$$


in which, $A_{r}$ is the road roughness coefficient $\left(\mathrm{m}^{3} /\right.$ cycle) representing the road roughness condition; $\bar{\phi}$ is the wave number (cycle/m); $\bar{\phi}_{0}$ is the discontinuity frequency $(0.5 \pi \mathrm{cycle} / \mathrm{m})$. The road surface roughness $r(x)$ can be generated by the inverse Fourier Transformation as shown in Eq. (4).

$$
r(x)=\sum_{i=1}^{n} \sqrt{2 S\left(\bar{\phi}_{k}\right) \Delta \bar{\phi}} \cos \left(2 \pi \bar{\phi}_{k} x+\theta_{k}\right)
$$

in which, $n$ is the number of points in the inverse Fourier Transform; $x$ is the location on the road surface; $\theta_{k}$ is the random phase angle with a uniform distribution between 0 and $2 \pi$. The dynamic forces between the $i^{\text {th }}$ vehicle and the contacting element on the bridge due to the road surface roughness can be expressed in the following equation:

$$
F_{r}^{i}(t)=\sum_{j=1}^{n_{a}}\left(K_{l z L}^{j} r\left(x_{j}\right)+C_{l z L}^{j} \dot{r}\left(x_{j}\right) V_{i}+K_{l z R}^{j} r\left(x_{j}\right)+C_{l z R}^{j} \dot{r}\left(x_{j}\right) V_{i}\right)
$$

in which, $r\left(x_{j}\right)$ is the road surface roughness at the $j^{\text {th }}$ axle; $\dot{r}\left(x_{j}\right)$ is the first order derivative of $r\left(x_{j}\right)$ with respect to time; $V_{i}$ is the driving speed of the $i^{\text {th }}$ vehicle; $n_{a}$ is the number of wheel axles.

\subsection{Modeling of wind forces on bridge}

The total wind forces acting on the bridge are commonly divided into three components: steady-state forces resulting from the average wind speed component, self-excited forces resulting from the bridgewind interactions and buffeting forces resulting from the unsteady wind velocity component.

\subsubsection{Steady-state wind forces on bridge}

The steady-state wind forces acting on the bridge deck are divided into three directions, which are drag force, lift force and twist moment, as defined in Eqs. (6a-c), respectively.

$$
D=\frac{1}{2} \rho U_{w}^{2} C_{d}(\alpha) H
$$




$$
\begin{aligned}
& L=\frac{1}{2} \rho U_{w}^{2} C_{l}(\alpha) H \\
& M=\frac{1}{2} \rho U_{w}^{2} C_{m}(\alpha) B^{2}
\end{aligned}
$$

in which, $\rho$ is the mass density of the air; $U_{w}$ is the steady-state wind speed; $H$ is the depth of the bridge girder; $B$ is the width of the bridge girder; $\alpha$ is the effective wind attack angle, which is obtained by summing the initial wind attack angle and the rotational displacement of the bridge deck; $C_{d}(\alpha), C_{l}(\alpha)$ and $C_{m}(\alpha)$ are the static wind coefficients for the bridge girder, respectively, which change with the effective wind attack angle.

\subsubsection{Self-excited wind forces on bridge}

The components of the self-excited forces can be obtained by summing the response associated with the structural motion in lateral, vertical and torsional directions. Particularly, the self-excited drag force, lift force and twist moment on a unit span of bridge girder can be expressed as the convolution integral between the time-dependent bridge girder motion and the impulse functions associated with the motion, as shown in Eq. (7) (Lin and Yang 1983).

$$
\begin{aligned}
& D_{s e}(t)=D_{s e p}(t)+D_{s e h}(t)+D_{s e \alpha}(t)=\int_{-\infty}^{t} f_{D p}(t-\tau) p(\tau) d \tau+\int_{-\infty}^{t} f_{D h}(t-\tau) h(\tau) d \tau+\int_{-\infty}^{t} f_{D \alpha}(t-\tau) \alpha(\tau) d \tau \\
& L_{s e}(t)=L_{s e p}(t)+L_{s e h}(t)+L_{s e \alpha}(t)=\int_{-\infty}^{t} f_{L p}(t-\tau) p(\tau) d \tau+\int_{-\infty}^{t} f_{L h}(t-\tau) h(\tau) d \tau+\int_{-\infty}^{t} f_{L \alpha}(t-\tau) \alpha(\tau) d \tau \\
& M_{s e}(t)=M_{\text {sep }}(t)+M_{\text {seh }}(t)+M_{\text {se } \alpha}(t)=\int_{-\infty}^{t} f_{M p}(t-\tau) p(\tau) d \tau+\int_{-\infty}^{t} f_{M h}(t-\tau) h(\tau) d \tau+\int_{-\infty}^{t} f_{M \alpha}(t-\tau) \alpha(\tau) d \tau
\end{aligned}
$$

in which, $p(t), h(t)$ and $\alpha(t)$ are time-dependent lateral, vertical and rotational displacement responses of the bridge girder, respectively; $f_{D p}(t), f_{D h}(t), f_{D \alpha}(t), f_{L p}(t), f_{L h}(t), f_{L \alpha}(t), f_{M p}(t), f_{M h}(t)$ and $f_{M \alpha}(t)$ are the impulse response functions of the self-excited wind force components; The first subscripts " $D$ ", " $L$ " and " $M$ " define the impulse response functions corresponding to the self-excited drag, lift and twist forces, 
respectively; The second subscripts " $p$ ", " $h$ " and " $\alpha$ " define the impulse response function with respect to lateral, vertical and torsional unit impulse displacement, respectively.

The impulse response functions are evaluated by the experimentally determined flutter derivatives by means of a rational function approximation approach (Lin and Yang 1983). For each impulse response function, the corresponding frequency response function will be expressed using rational functions. The frequency-independent parameters are determined using least square fitting of flutter derivatives. After the frequency response functions are determined, the impulse response function can be obtained through the Fourier Transform for each motion.

\subsubsection{Buffeting forces on bridge}

Buffeting forces on the bridge girder are induced by the unsteady components of the oncoming wind, including the time-variant horizontal and vertical parts. The buffeting forces on a unit span of bridge girder can be expressed as the convolution integral between the wind fluctuations and the associated impulse response functions in both horizontal and vertical directions using the similar way to that formulating the self-excited forces. The drag force, lift force and twist moment of buffeting forces can be expressed as:

$$
\begin{aligned}
& D_{b}(t)=D_{b u}(t)+D_{b w}(t)=\int_{-\infty}^{t} f_{D u}(t-\tau) u(\tau) d \tau+\int_{-\infty}^{t} f_{D w}(t-\tau) w(\tau) d \tau \\
& L_{b}(t)=L_{b u}(t)+L_{b w}(t)=\int_{-\infty}^{t} f_{L u}(t-\tau) u(\tau) d \tau+\int_{-\infty}^{t} f_{L w}(t-\tau) w(\tau) d \tau \\
& M_{b}(t)=M_{b u}(t)+M_{b w}(t)=\int_{-\infty}^{t} f_{M u}(t-\tau) u(\tau) d \tau+\int_{-\infty}^{t} f_{M w}(t-\tau) w(\tau) d \tau
\end{aligned}
$$

in which, $u(t)$ and $w(t)$ are the turbulent wind velocities in the horizontal and vertical directions, respectively; $f_{D u}(t), f_{D w}(t), f_{L u}(t), f_{L w}(t), f_{M u}(t)$ and $f_{M w}(t)$ are the impulse response functions; The subscripts " $D$ ", " $L$ " and " $M$ " denote the impulse response functions corresponding to drag force, lift force and twist moment, respectively; The subscripts " $u$ " and " $w$ " define the impulse response functions with 
respect to turbulent wind velocities in the horizontal and vertical directions, respectively. Similar to the approach used in the self-excited forces, the buffeting forces can be formulated in the time domain by means of the frequency-dependent aerodynamic admittance functions.

\subsection{Modeling of wind forces on vehicles}

The total wind forces acting on a road vehicle are determined using the quasi-static approach (Baker 1986). The instantaneous wind speed on the vehicle is obtained as the summation of mean wind speed $U_{w}$ and unsteady horizontal wind speed $u(x, t)$ at location $x$ and time $t$. The road vehicles on the road or bridge have varying driving speeds following some traffic rules and the instantaneous vehicle driving speed is defined as $U_{v e}(t)$ at time $t$. The wind velocity relative to the vehicle $U_{R}(t)$ at time $t$ is obtained from the vector subtraction of $U_{v e}(t)$ from $\left(U_{w}+u(x, t)\right)$, as shown in Fig. 3.

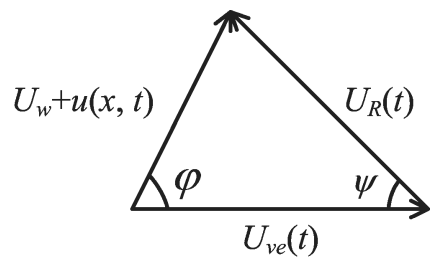

Fig. 3 Demonstration of the relative wind speed

The wind speed relative to the vehicle $U_{R}(t)$ can be obtained in Eq. (9a). $\varphi$ is the wind attack angle, which is the angle between the wind and vehicle driving directions. $\psi$ is the yaw angle, which is the angle between the direction of the relative wind speed and the vehicle driving direction in the range from 0 to $\pi$, as defined in Eq. (9b).

$$
\begin{gathered}
U_{R}(t)=\sqrt{\left(U_{w}+u(x, t)\right)^{2}+U_{v e}^{2}(t)-2 U_{v e}\left(U_{w}+u(x, t)\right) \cos \varphi} \\
\psi=\arctan \left[\frac{\left(U_{w}+u(x, t)\right) \sin \varphi}{U_{v e}(t)-\left(U_{w}+u(x, t)\right) \cos \varphi}\right]
\end{gathered}
$$

The total wind forces and moments on the vehicles are given in the following equations: 


$$
\begin{aligned}
& F_{v x}^{t}=\frac{1}{2} \rho U_{R}^{2}(t) C_{D}(\Psi) A \quad(10 \mathrm{a}) \quad F_{v y}^{t}=\frac{1}{2} \rho U_{R}^{2}(t) C_{S}(\Psi) A \quad(10 \mathrm{~b}) \quad F_{v z}^{t}=\frac{1}{2} \rho U_{R}^{2}(t) C_{L}(\Psi) A \\
& M_{v x}^{t}=\frac{1}{2} \rho U_{R}^{2}(t) C_{R}(\Psi) A h_{v}(10 \mathrm{~d}) \quad M_{v y}^{t}=\frac{1}{2} \rho U_{R}^{2}(t) C_{P}(\Psi) A h_{v} \quad(10 \mathrm{e}) \quad M_{v z}^{t}=\frac{1}{2} \rho U_{R}^{2}(t) C_{Y}(\Psi) A h_{v}
\end{aligned}
$$

in which, $F_{v x}^{w}, F_{v y}^{w}$ and $F_{v z}^{w}$ are the total drag force, side force and lift force acting at the center of gravity in the positive $x, y$ and $z$ direction, respectively; $M_{v x}^{w}, M_{v y}^{w}$ and $M_{v z}^{w}$ are the total rolling moment, pitching moment and yawing moment about the $x, y$ and $z$ directions, respectively; $C_{D}(\Psi), C_{S}(\Psi), C_{L}(\Psi)$, $C_{R}(\Psi), C_{P}(\Psi)$ and $C_{Y}(\Psi)$ are the coefficients of drag force, side force, lift force, rolling moment, pitching moment and yawing moment, respectively; $A$ is the reference area, which is usually taken as the frontal area of the vehicle; $h_{v}$ is the reference height, which is usually taken as the distance between the gravity of the vehicle and the ground. The direction of each wind force component on the vehicle is demonstrated in Fig. 4. The positive driving direction is in the positive $x$ direction and wind is assumed to blow in the positive $y$ direction.

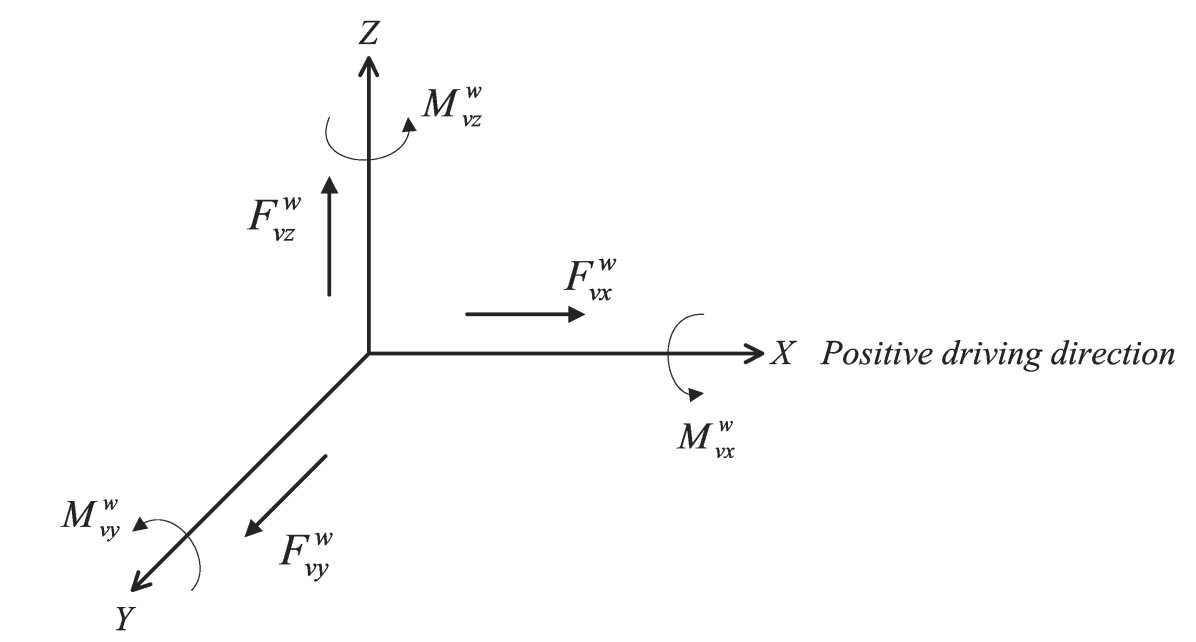

Wind direction

Fig. 4 Demonstration of the direction of each wind force component on the vehicle

The total wind forces on the vehicle consist of the effects from both mean wind speed and unsteady wind speed, and therefore include the combined static and dynamic effects. In the coupled bridge-traffic 
interaction analysis, only the unsteady part of the wind forces contributes to the dynamic coupled analysis between the bridge and vehicles. In this study, the total wind forces on vehicles are partitioned into two parts, which are the static wind force and the aerodynamic wind force. The static wind forces in each direction only consider the static wind effects from the mean wind speed $U_{w}$ and exclude the contribution of the unsteady wind speed $u(x, t)$ and vehicle speed $U_{v e}(t)$ that change with respect to time. The static wind forces are used in the nonlinear static analysis to identify the deformed position for the following nonlinear dynamic analysis. The static wind forces and moments in each direction are expressed as follows:

$$
\begin{aligned}
& F_{v x}^{s}=\frac{1}{2} \rho U_{w}{ }^{2} C_{D}\left(\Psi^{\prime}\right) A \\
& M_{v x}^{s}=\frac{1}{2} \rho U_{w}{ }^{2} C_{R}\left(\Psi^{\prime}\right) A h_{v} \\
& F_{v y}^{s}=\frac{1}{2} \rho U_{w}{ }^{2} C_{S}\left(\Psi^{\prime}\right) A \\
& F_{v z}^{s}=\frac{1}{2} \rho U_{w}{ }^{2} C_{L}\left(\Psi^{\prime}\right) A \\
& M_{v y}^{s}=\frac{1}{2} \rho U_{w}{ }^{2} C_{P}\left(\Psi^{\prime}\right) A h_{v} \\
& M_{v z}^{s}=\frac{1}{2} \rho U_{w}{ }^{2} C_{Y}\left(\Psi^{\prime}\right) A h_{v}
\end{aligned}
$$

In which, $\Psi$ is the angle between the wind speed and the longitudinal direction of the bridge. The static wind force on a vehicle does not consider the effect of vehicle motion. The aerodynamic wind force on a vehicle in each direction is obtained by subtracting static wind force from the total wind force, as shown in Eq. (12).

$$
\begin{aligned}
& F_{v x}^{d}=F_{v x}^{t}-F_{v x}^{s} \quad(12 \mathrm{a}) \quad F_{v y}^{d}=F_{v y}^{t}-F_{v y}^{s} \quad(12 \mathrm{~b}) \quad F_{v z}^{d}=F_{v z}^{t}-F_{v z}^{s} \\
& M_{v x}^{d}=M_{v x}^{t}-M_{v x}^{s} \quad(12 \mathrm{~d}) \quad M_{v y}^{d}=M_{v y}^{t}-M_{v y}^{s} \quad(12 \mathrm{e}) \quad M_{v z}^{d}=M_{v z}^{t}-M_{v z}^{s}
\end{aligned}
$$

The drag force can hardly cause an accident on the vehicle because the driver usually can adjust the traction to keep the driving speed and resist the drag force. Since the vehicle model doesn't have the corresponding degree of freedom for the yawing moment, the equivalent lateral forces on the front and rear wheels are obtained based on the force equilibrium conditions in order to resist the yawing moment. 


\section{Dynamic bridge-traffic-wind interaction analysis}

\subsection{Simulation of stochastic traffic flow}

The stochastic traffic flow in this study is simulated using the cellular automaton (CA) model to rationally simulate the moving traffic on the long-span bridge. The discrete variables of an individual vehicle in the two-lane traffic flow simulated in the present study include the vehicle-occupied lane, vehicle longitudinal location, vehicle type, vehicle speed, vehicle driving direction. The variables in each cellular are updated based on the vehicle information in the adjacent locations and the probabilistic traffic rules regulating the accelerating, decelerating, lane changing and braking operations. Detailed traffic rules involved in the traffic flow simulation are referred to the published papers (Chen and $\mathrm{Wu} 2010$; Chen and Wu 2011). The CA-based traffic flow simulation is performed on a roadway-bridge-roadway system to simulate the stochastic traffic flow through the bridge in a realistic way. The periodic boundary conditions are adopted in the simulation of stochastic traffic flow such that the number of each type of vehicles in the roadway-bridge-roadway system remains the same.

\subsection{Equations of motion for the bridge-traffic system}

The fully-coupled bridge-traffic interaction system is established with the bridge and vehicles as two subsystems. The bridge subsystem is built based on the finite element formulation of the bridge and the vehicle subsystem is built based on the numerical dynamic model of each vehicle. The interaction forces between the bridge and vehicles are formulated in terms of the coupling matrices and motion vectors of the bridge and each vehicle on the bridge. It is assumed that the two approaching roadways are completely rigid for both driving directions, and therefore the vehicle vibrates on its own without the interaction between the vehicle and supporting road surface when the vehicle is on the approaching roads. The coupled motion equations of the bridge-traffic system can be constructed as in Eq. (13). 


$$
\begin{aligned}
& {\left[\begin{array}{cccc}
M_{b} & 0 & \cdots & 0 \\
0 & M_{v_{1}} & \cdots & 0 \\
\vdots & \vdots & \ddots & \vdots \\
0 & 0 & \cdots & M_{v_{n}}
\end{array}\right]\left\{\begin{array}{c}
\ddot{q}_{b} \\
\ddot{q}_{v_{1}} \\
\vdots \\
\ddot{q}_{v_{n}}
\end{array}\right\}+\left[\begin{array}{cccc}
C_{b} & 0 & \cdots & 0 \\
0 & C_{v_{1}} & \cdots & 0 \\
\vdots & \vdots & \ddots & \vdots \\
0 & 0 & 0 & C_{v_{n}}
\end{array}\right]\left\{\begin{array}{c}
\dot{q}_{b} \\
\dot{q}_{v_{1}} \\
\vdots \\
\dot{q}_{v_{n}}
\end{array}\right\}+\left[\begin{array}{cccc}
K_{b} & 0 & \cdots & 0 \\
0 & K_{v_{1}} & \cdots & 0 \\
\vdots & \vdots & \ddots & \vdots \\
0 & 0 & \cdots & K_{v_{n}}
\end{array}\right]\left\{\begin{array}{c}
q_{b} \\
q_{v_{1}} \\
\vdots \\
q_{v_{n}}
\end{array}\right\}} \\
& =\left\{\begin{array}{c}
\sum_{i=1}^{n} F_{v_{i}}^{G}+F_{b}{ }^{R}+F_{b}^{C}+F_{b}^{S e}+F_{b}^{B u} \\
F_{v_{1}}{ }^{B}+F_{v_{1}}^{C}+F_{v_{1}}^{d w} \\
\vdots \\
F_{v_{n}}{ }^{R}+F_{v_{n}}{ }^{C}+F_{v_{n}}{ }^{d w}
\end{array}\right\}
\end{aligned}
$$

in which,

$$
\begin{gathered}
F_{b}^{C}=\sum_{i=1}^{n} C_{b c i}\left\{\dot{q}_{b}\right\}+\sum_{i=1}^{n} K_{b c i}\left\{q_{b}\right\}+\sum_{i=1}^{n} C_{b, v_{i}}\left\{\dot{q}_{v_{i}}\right\}+\sum_{i=1}^{n} K_{b, v_{i}}\left\{q_{v_{i}}\right\} \\
F_{v_{i}}{ }^{C}=C_{v_{i}, b}\left\{\dot{q}_{v_{i}}\right\}+K_{v_{i}, b}\left\{q_{v_{i}}\right\}
\end{gathered}
$$

$q_{b}$ and $q_{v_{i}}$ are the displacement vectors of the bridge and the $i^{\text {th }}$ vehicle, respectively; one-dot and two-dot superscripts of the displacement vector indicate the corresponding velocity and acceleration, respectively; $n$ is the total number of vehicles involved in the traffic flow; $M_{b\left(v_{i}\right)}, K_{b\left(v_{i}\right)}$ and $C_{b\left(v_{i}\right)}$ are the structural mass, stiffness and damping matrices for the bridge subsystem ( $i^{\text {th }}$ vehicle); $K_{b c i}$ and $C_{b c i}$ refer to the stiffness and damping contributions to the bridge structure due to the coupling effects between the $i^{\text {th }}$ vehicle in the traffic flow and the bridge system, respectively; $K_{b, v_{i}}$ and $C_{b, v_{i}}$ are the coupled stiffness and damping matrices contributing to the bridge vibration from the $i^{\text {th }}$ vehicle in the traffic flow, respectively; $K_{v_{i}, b}$ and $C_{v_{i}, b}$ are the coupled stiffness and damping matrices contributing to the vibration of the $i^{\text {th }}$ vehicle in the traffic flow from the bridge structure; $F_{b}$ and $F_{v_{i}}$ denote the external force vector due to the applied loads for the bridge and the $i^{\text {th }}$ vehicle, respectively; the superscripts $R$ and $C$ indicate the interaction forces due to road surface roughness and coupling matrices on the bridge or each vehicle, respectively; the superscripts $G, S e$ and $B u$ represent for the external loads from vehicle gravity, self-excited and 
buffeting forces on the bridge, respectively; superscript $d w$ indicates the aerodynamic wind force on vehicles.

The structural matrices for the vehicles and the coupling matrices between the bridge and each vehicle are derived using the virtual work approach (Cai and Chen, 2004). The coupling matrices between the bridge and each vehicle are updated at every time step during the simulation process based on the new contact location of each vehicle traveling on the bridge. The self-excited forces due to the bridge-structure interactions and coupling interaction forces between the bridge and vehicles are motion-dependent, and thus are unknown at the beginning of each time step. The nonlinear iteration is therefore undertaken starting with an initial motion vector until the prescribed convergence criterion is satisfied at each time step.

\section{Vehicle accident analysis}

\subsection{The total force approach}

The dynamic interaction analysis between the bridge and vehicles assumes that the vehicle wheels and the bridge deck surface have point contact at all times and the wheels will not be separated from the bridge during the simulation. Based on the nonlinear finite element model, the total contact forces in the vertical and lateral directions can be obtained for the vehicles throughout the analysis. The total contact forces in the vertical and lateral directions for each wheel at one side at time $t$ are noted as $V_{i L(R)}^{t}(t)$ and $L_{i L(R)}^{t}(t)(i=$ $\left.1,2, \ldots, n_{a}\right)$, respectively. These total contact forces are obtained through following steps:

Step 1: Nonlinear static analysis is conducted for the bridge under bridge gravity, initial pretension forces of the cables and static wind forces on the bridge. This step generates the initial deformed position of the bridge for following nonlinear dynamic analysis.

Step 2: Static analysis is conducted for the vehicles under vehicle gravity and static wind forces on vehicles. The initial deformed position of each vehicle on the bridge will be generated and the static 
vertical and lateral contact forces $V_{i L(R)}^{s}$ and $L_{i L(R)}^{s}$ are obtained as the summation of the forces of the lower spring and damper on the left (right) side of the $i^{\text {th }}$ wheel set, respectively, as shown in Eq. (14).

$$
\begin{gathered}
V_{i L(R)}^{s}=K_{l z L(R)}^{i} h_{L(R)}^{i}+C_{l z L(R)}^{i} \dot{h}_{L(R)}^{i} \\
L_{i L(R)}^{s}=K_{l y L(R)}^{i} y_{L(R)}^{i}+C_{l y L(R)}^{i} \dot{y}_{L(R)}^{i}
\end{gathered}
$$

in which, $K_{l z L(R)}^{i}$ and $C_{l z L(R)}^{i}$ are the stiffness and damping coefficients of the lower vertical spring and damper of the $i^{\text {th }}$ wheel set on the left (right) side; $K_{l y L R)}^{i}$ and $C_{l y L(R)}^{i}$ are the stiffness and damping coefficients of the lower lateral spring and damper of the $i^{\text {th }}$ wheel set on the left (right) side; $h_{L(R)}^{i}$ and $\dot{h}_{L(R)}^{i}$ are the vertical displacement and velocity of the mass block of the $i^{\text {th }}$ wheel set on the left (right) side, respectively; $y_{L(R)}^{i}$ and $\dot{y}_{L(R)}^{i}$ are the lateral displacement and velocity of the mass block of the $i^{\text {th }}$ suspension and wheel set on the left (right) side, respectively.

Step 3: Starting from the deformed position of the bridge and vehicles, nonlinear dynamic analysis is conducted for the bridge-traffic system, in which the aerodynamic forces on the bridge, vehicles and the interaction forces between the bridge and vehicles are applied.

In this step, the displacement and velocity of the mass block relative to the bridge at each contact point need to be obtained in order to calculate the forces in the lower spring and damper of the wheel set. Considering that the dynamic displacement and velocity of the vehicle and the bridge are unknown at first in each time step, nonlinear iterations are conducted starting from the initial deformed position. The dynamic total contact force $V_{i L(R)}^{d}$ and $L_{i L(R)}^{d}$ in the vertical and lateral directions for each time step are expressed in Eq. (15).

$$
V_{i L(R)}^{d}(t)=K_{l z L(R)}^{i} \bar{h}_{L(R)}^{i}(t)+C_{l z L(R)}^{i} \overline{\dot{h}}_{L(R)}^{i}(t)
$$




$$
L_{i L(R)}^{d}(t)=K_{l y L(R)}^{i} \bar{y}_{L(R)}^{i}(t)+C_{l y L(R)}^{i} \overline{\dot{y}}_{L(R)}^{i}(t)
$$

in which, $\bar{h}_{L(R)}^{i}(t)$ and $\overline{\dot{h}}_{L(R)}^{i}(t)$ are the vertical displacement and velocity of the mass block relative to the bridge contact point for the $i^{\text {th }}$ wheel set on the left (right) side at time $t$, respectively; $\bar{y}_{L(R)}^{i}(t)$ and $\overline{\dot{y}}_{L(R)}^{i}(t)$ are the lateral displacement and velocity of the mass block relative to the bridge contact point for the $i^{\text {th }}$ suspension and wheel set on the left (right) side at time $t$, respectively.

Step 4: The total vertical $\left(V_{i L(R)}^{t}(t)\right)$ and lateral $\left(L_{i L(R)}^{t}(t)\right)$ contact force time histories are obtained as the summation of the static and dynamic vertical and lateral contact forces at each time instant, respectively, as shown in Eq. (16).

$$
\begin{aligned}
& V_{i L(R)}^{t}(t)=V_{i L(R)}^{s}+V_{i L(R)}^{d}(t) \\
& L_{i L(R)}^{t}(t)=L_{i L(R)}^{s}+L_{i L(R)}^{d}(t)
\end{aligned}
$$

\subsection{Characterization of vehicle accident type}

After the time histories of vertical and lateral contact forces at each wheel set are obtained, the vehicle accident analysis is carried out based on the vehicle mechanics theory. The proposed total force approach is able to identify the three vehicle accident types: lift-up, side-slip and yawing.

\subsubsection{Type I: Lift-up at the wheel}

Lift-up accident is defined as the situation when at least one side of a certain wheel set is lifted up- losing contact with the ground. It is known that the lift-up of wheel may or may not eventually lead to overturning accidents. However, given the complexness and uncertainties of the time-transient overturning process (Chen and Chen 2010), lift-up accident criterion can be used as an ideal and convenient evaluation tool to assess the associated risks conservatively. Based on the coordinates defined in this study, lift-up accidents are considered to happen once the total vertical contact force on the ground 
at one side of one or all wheel sets at certain time instants are over zero. Lift-up accidents for a high-sided heavy truck may include three situations, such as lift-up occurring at one wheel set, two adjacent wheel sets and all wheel sets. For a 3-axle heavy truck, the accident criterion is shown below.

1) Lift-up at $i^{\text {th }}$ wheel axle: $V_{i L(R)}^{t}(t)>0, i=1,2$ or 3

2) Lift-up at two adjacent wheel axles: $V_{i L(R)}^{t}(t)>0, i=1$ and $2 ; 2$ and 3

3) Lift-up at all wheel axles: $V_{i L(R)}^{t}(t)>0, i=1,2$ and 3

When the lift-up accident happens to one wheel set, two wheel sets or all the wheel sets, the vehicle may start to rotate about the contact points of the wheels remaining on the ground. The lift-up process may eventually trigger overturning accidents, and therefore should be prevented for the safety of the driver as well as the vehicle. Existing research has shown that lift-up accidents are the most common type for high-sided trucks in the windy environment (e.g. Chen and Cai 2004; Chen and Chen 2010).

\subsubsection{Type II: Side-slip at the wheel}

A side-slip accident is defined as the situation when a certain wheel remains contact on the ground whereas the lateral friction cannot prevent the wheels from sliding laterally. When the left and right wheels of a certain wheel set both remain contact on the ground, the side-slip accident will happen if the total lateral contact force of the left and right wheel exceeds the total static friction force. The static friction force is the multiplication of static friction coefficient $\mu$ and the vertical contact force. Similar to overturning accidents, side-slip accidents for a high-sided heavy truck also have three possible situations: side-slip at one wheel set, at two adjacent wheel sets and at all wheel sets. The corresponding accident criteria for heavy trucks are shown as follows:

1) Side-slip at $i^{\text {th }}$ wheel axle: $\left|L_{i L}^{t}(t)+L_{i R}^{t}(t)\right|>\mu\left|V_{i L}^{t}(t)+V_{i R}^{t}(t)\right|, i=1,2$ or 3

2) Side-slip at two adjacent wheel axles: $\left|L_{i L}^{t}(t)+L_{i R}^{t}(t)\right|>\mu\left|V_{i L}^{t}(t)+V_{i R}^{t}(t)\right|, i=1$ and 2; or $i=2$ and 3 
3) Side-slip at all wheel axles: $\left|L_{i L}^{t}(t)+L_{i R}^{t}(t)\right|>\mu\left|V_{i L}^{t}(t)+V_{i R}^{t}(t)\right|, i=1,2$ and 3

When the side-slip accident happens along with lift-up accidents at certain wheel sets, the vertical contact force at the lifted side of the wheel set should be set to zero for the above criteria. The criteria for the car and light truck can be easily derived from above based on the actual axle number.

\subsubsection{Type III: Yawing accident (rotational movement)}

A yawing accident is defined as the situation when the vehicle has rotational movements around the vertical axis. It could happen when all or only some wheels experience side-slipping. Particularly, the yawing accident for a heavy truck can happen in terms of rotating between $1^{\text {st }}$ and $2^{\text {nd }}$ wheel sets or

between $2^{\text {nd }}$ and $3^{\text {rd }}$ wheel sets. The accident criterion for each situation of a heavy truck is shown as follows.

1) Yawing around vertical axis between $i^{\text {th }}$ and $j^{\text {th }}$ wheel sets when side-slip occurs to all wheels:

$\operatorname{sign}\left(L_{i L}^{t}(t)+L_{i R}^{t}(t)\right) \neq \operatorname{sign}\left(L_{j L}^{t}(t)+L_{j R}^{t}(t)\right), i=1, j=2$ or $i=2, j=3$,

and

$\left|L_{i L}^{t}(t)+L_{i R}^{t}(t)\right|>\mu\left|V_{i L}^{t}(t)+V_{i R}^{t}(t)\right|, i=1,2$ and 3,

2) Yawing around vertical axis between $i^{\text {th }}$ and $j^{\text {th }}$ wheel sets when side-slip doesn't happen to all:

$\left|L_{i L}^{t}(t)+L_{i R}^{t}(t)\right|>\mu\left|V_{i L}^{t}(t)+V_{i R}^{t}(t)\right|, i=1,2$ or 3

and

$\left|L_{j L}^{t}(t)+L_{j R}^{t}(t)\right| \leq \mu\left|V_{j L}^{t}(t)+V_{j R}^{t}(t)\right|, j=1,2$ or 3 and $j \neq i$

3) Yawing around vertical axis at the pivot connecting two rigid bodies:

$\left|L_{i L}^{t}(t)+L_{i R}^{t}(t)\right| \leq \mu\left|V_{i L}^{t}(t)+V_{i R}^{t}(t)\right|, i=1,2 ; L_{3 L}^{t}(t)+L_{3 R}^{t}(t)>\mu\left(V_{3 L}^{t}(t)+V_{3 R}^{t}(t)\right)$, 
Or,

$\left|L_{i L}^{t}(t)+L_{i R}^{t}(t)\right|>\mu\left|V_{i L}^{t}(t)+V_{i R}^{t}(t)\right|, i=1,2 ; L_{3 L}^{t}(t)+L_{3 R}^{t}(t) \leq \mu\left(V_{3 L}^{t}(t)+V_{3 R}^{t}(t)\right)$

Yawing accidents for light trucks and sedan cars only have the first two types of situations as illustrated above.

\section{Numerical demonstration}

\subsection{Prototype bridge and vehicle models}

The prototype cable-stayed bridge in the present study has a total length of $836.7 \mathrm{~m}$, with a main span, two side spans and two approach spans as shown in Fig. 5. The cable-stayed bridge has a bridge deck with a steel twin-box cross-section, which has a width of $28 \mathrm{~m}$ and a height of $4.57 \mathrm{~m}$. The two steel pylons have A-shaped cross-sections with a height of $103.6 \mathrm{~m}$. The bridge superstructure is supported by the cross beam of bridge pylons at the pylon locations and the reinforced concrete bridge piers at the side spans with longitudinally sliding bearing supports.

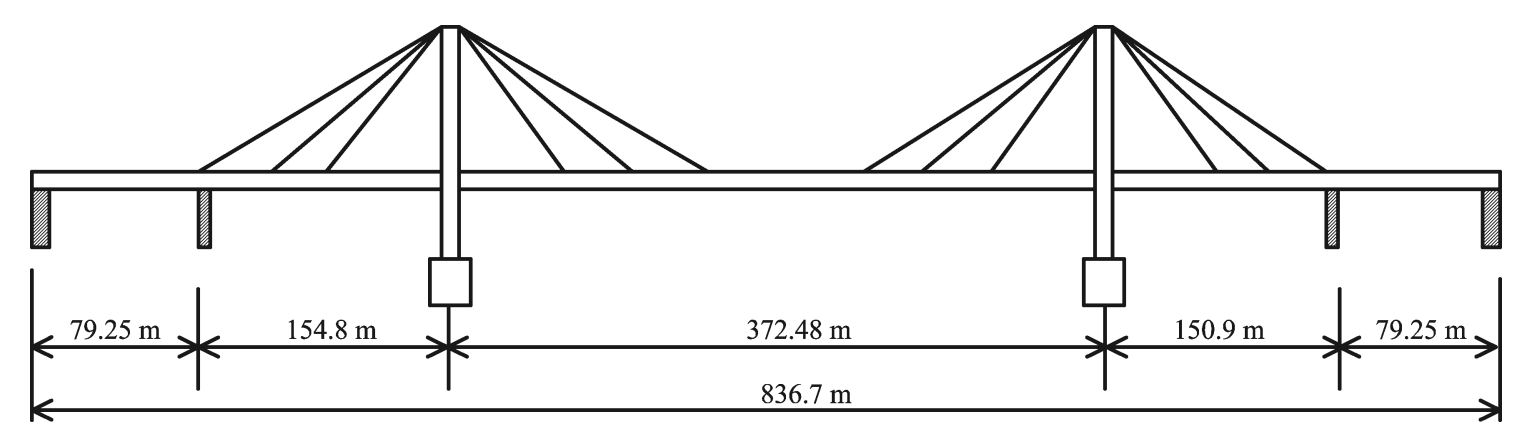

Fig. 5 Elevation view of the long-span cable-stayed bridge

The travelling vehicles in this study are classified into three types: heavy truck, light truck and light sedan car. The dynamic parameters for each type of vehicles involved in the present study, including mass, 
mass moment of inertia, stiffness coefficients and damping coefficients, are listed in Table 1. The dimension parameters for each type of vehicles are listed in Table 2.

Table 1 Dynamic parameters of the vehicles used in the case study

\begin{tabular}{|c|c|c|c|c|}
\hline Parameter & Unit & Heavy truck & Light truck & Sedan car \\
\hline Mass of rigid body $1\left(M_{r}^{1}\right)$ & $k g$ & 4000 & 6500 & 1600 \\
\hline Pitching moment of inertia of rigid body $1\left(J_{y r}^{1}\right)$ & $\mathrm{kg} \cdot \mathrm{m}^{2}$ & 10500 & 9550 & 1850 \\
\hline Rolling moment of inertia of rigid body $1\left(J_{x r}^{1}\right)$ & $\mathrm{kg} \cdot \mathrm{m}^{2}$ & 3200 & 3030 & 506 \\
\hline Mass of rigid body $2\left(M_{r}^{2}\right)$ & $k g$ & 12500 & - & - \\
\hline Pitching moment of inertia of rigid body $2\left(J_{y r}^{2}\right)$ & $\mathrm{kg} \cdot \mathrm{m}^{2}$ & 28500 & - & - \\
\hline Rolling moment of inertia of rigid body $2\left(J_{x r}^{2}\right)$ & $\mathrm{kg} \cdot \mathrm{m}^{2}$ & 10500 & - & - \\
\hline Mass of axle block $1\left(M_{a L}^{1}, M_{a R}^{1}\right)$ & $\mathrm{kg}$ & 370 & 800 & 39.5 \\
\hline Mass of axle block $2\left(M_{a L}^{2}, M_{a R}^{2}\right)$ & $k g$ & 1250 & 800 & 39.5 \\
\hline Mass of axle block $3\left(M_{a L}^{3}, M_{a R}^{3}\right)$ & $k g$ & 1100 & - & - \\
\hline Upper vertical spring stiffness $\left(K_{u z L}^{1}, K_{u z R}^{1}\right)$ & $N / m$ & 100 & 250 & 109 \\
\hline Upper vertical spring stiffness $\left(K_{u z L}^{2}, K_{u z R}^{2}\right)$ & $N / m$ & 250 & 250 & 109 \\
\hline Upper vertical spring stiffness $\left(K_{u z L}^{3}, K_{u z R}^{3}\right)$ & $N / m$ & 400 & - & - \\
\hline Lower vertical spring stiffness $\left(K_{l z L}^{1}, K_{l z R}^{1}\right)$ & $N / m$ & 150 & 175 & 176 \\
\hline Lower vertical spring stiffness $\left(K_{l z L}^{2}, K_{l z R}^{2}\right)$ & $N / m$ & 400 & 175 & 176 \\
\hline Lower vertical spring stiffness $\left(K_{l z L}^{3}, K_{l z R}^{3}\right)$ & $N / m$ & 500 & - & - \\
\hline Upper lateral spring stiffness $\left(K_{u y L}^{1}, K_{u y R}^{1}\right)$ & $N / m$ & 75 & 187.5 & 79.5 \\
\hline
\end{tabular}




\begin{tabular}{|c|c|c|c|c|}
\hline Parameter & Unit & Heavy truck & Light truck & Sedan car \\
\hline Upper lateral spring stiffness $\left(K_{u y L}^{2}, K_{u y R}^{2}\right)$ & $N / m$ & 187.5 & 187.5 & 79.5 \\
\hline Upper lateral spring stiffness $\left(K_{u y L}^{3}, K_{u y R}^{3}\right)$ & $N / m$ & 300 & - & - \\
\hline Lower lateral spring stiffness $\left(K_{l y L}^{1}, K_{l y R}^{1}\right)$ & $N / m$ & 72 & 100 & 58.7 \\
\hline Lower lateral spring stiffness $\left(K_{l y L}^{2}, K_{l y R}^{2}\right)$ & $N / m$ & 131 & 100 & 58.7 \\
\hline Lower lateral spring stiffness $\left(K_{l y L}^{3}, K_{l y R}^{3}\right)$ & $N / m$ & 167 & - & - \\
\hline \multicolumn{5}{|l|}{ Upper vertical/lateral damping coefficient } \\
\hline$\left(C_{u z L}^{1}, C_{u z R}^{1}, C_{u y L}^{1}, C_{u y R}^{1}\right)$ & $N \cdot s / m$ & 5 & 2.5 & 0.8 \\
\hline \multicolumn{5}{|l|}{ Upper vertical/lateral damping coefficient } \\
\hline$\left(C_{u z L}^{2}, C_{u z R}^{2}, C_{u y L}^{2}, C_{u y R}^{2}\right)$ & $N \cdot s / m$ & 30 & 2.5 & 0.8 \\
\hline \multicolumn{5}{|l|}{ Upper vertical/lateral damping coefficient } \\
\hline$\left(C_{u z L}^{3}, C_{u z R}^{3}, C_{u y L}^{3}, C_{u y R}^{3}\right)$ & $N \cdot s / m$ & 40 & - & - \\
\hline \multicolumn{5}{|l|}{ Lower vertical/lateral damping coefficient } \\
\hline$\left(C_{l z L}^{1}, C_{l z R}^{1}, C_{l y L}^{1}, C_{l y R}^{1}\right)$ & $N \cdot s / m$ & 1.2 & 1 & 0.8 \\
\hline \multicolumn{5}{|l|}{ Lower vertical/lateral damping coefficient } \\
\hline$\left(C_{l z L}^{2}, C_{l z R}^{2}, C_{l y L}^{2}, C_{l y R}^{2}\right)$ & $N \cdot s / m$ & 4.5 & 1 & 0.8 \\
\hline \multicolumn{5}{|l|}{ Lower vertical/lateral damping coefficient } \\
\hline$\left(C_{l z L}^{3}, C_{l z R}^{3}, C_{l y L}^{3}, C_{l y R}^{3}\right)$ & $N \cdot s / m$ & 4.5 & - & - \\
\hline
\end{tabular}

Table 2 Dimensions of the vehicles used in the case study 


\begin{tabular}{ccccc}
\hline Parameter & Unit & Heavy truck & Light truck & Light car \\
\hline Distance between axle 1 and rigid body 1 $\left(D_{l}\right)$ & $m$ & 1.83 & 2.8 & 1.34 \\
Distance between axle 2 and rigid body 1 $\left(D_{2}\right)$ & $m$ & 1.83 & 4.8 & 1.34 \\
Distance between axle 2 and rigid body 2 $\left(D_{3}\right)$ & $m$ & 3.60 & - & - \\
Distance between axle 3 and rigid body 2 $\left(D_{4}\right)$ & $m$ & 2.60 & - & - \\
Distance between pin and rigid body 1 $\left(D_{5}\right)$ & $m$ & 1.83 & - & - \\
Distance between pin and rigid body 2 $\left(D_{6}\right)$ & $m$ & 3.60 & - & 0.8 \\
Distance between left axle and rigid body 1 $\left(W_{1}\right)$ & $m$ & 1.10 & 1.0 & - \\
Distance between right axle and rigid body 1 $\left(W_{2}\right)$ & $m$ & 1.10 & - & 1.96 \\
Frontal area $(A)$ & $m$ & 10.5 & 7.5 & 1.1 \\
Reference height $\left(h_{v}\right)$ & $m$ & 2.0 & 1.85 & 11.6 \\
Total length of vehicle $(L)$ & $m$ & 13.4 & & 4.54
\end{tabular}

\subsection{Simulation of stochastic traffic flow}

The total length of the roadway-bridge-roadway path is $1,260 \mathrm{~m}$, including two roadways with a length of $210 \mathrm{~m}$ each and the bridge with a length of $840 \mathrm{~m}$. The speed limit is adopted as $30 \mathrm{~m} / \mathrm{s}$. The stochastic traffic flow with a moderate density of 16 vehicles $/ \mathrm{km} / \mathrm{lane}$ is simulated with the CA-based traffic flow simulation model as introduced previously (Chen and $\mathrm{Wu} 2011$ ). The percentages of the three types of vehicles in the traffic flow are chosen as $20 \%, 30 \%$ and $50 \%$ for heavy trucks, light trucks and light sedan cars, respectively. The simulated stochastic traffic flow is comprised of a total of 80 vehicles in the roadway-bridge-roadway system, including 16 heavy trucks, 24 light trucks and 40 light cars.

\subsection{Simulation of wind forces on bridge and vehicles}

The turbulent wind speeds are simulated as multi-variate stationary random Gaussian process with a mean value of zero in the vertical and lateral directions. In this study, wind speed is assumed to be 
perpendicular to the longitudinal axis of the bridge deck and the effects of other skewed wind directions are not considered. It is noted that the aerostatic and aerodynamic coefficients of the bridge in the present study adopt the original wind parameters of the bridge without considering wind interference between the bridge and moving vehicles. The reasons for choosing original static wind coefficients and flutter derivatives are primarily due to the lack of the state-of-the-art techniques to quantify wind coefficients of the coupled system of bridge and moving stochastic traffic flow. Although there have been some limited studies on wind tunnel testing with moving vehicles with assumed patterns (e.g., Han et al. 2014), no study has been reported in terms of the realistic stochastic traffic flow. It was found in the study by Han et al. (2014) that the effect of aerodynamic interference on the flutter derivatives of the bridge has little effects on the dynamic displacements of the bridge and the vehicle under service condition. In fact, given the stochastic nature of the traffic flow, the shape of the combined cross-section for the bridge-traffic system keeps varying over time, which poses serious challenges on rationally quantifying the aerodynamic interference effects.

Similar challenges as stated above exist for obtaining realistic wind coefficients for each traveling vehicle on the bridge. The aerodynamic interference for a specific vehicle exists not only between the bridge and vehicle but also exists due to vehicles that are passing by the designated vehicle. The wind coefficients may be influenced by the movements of the supporting structure and the surrounding moving vehicles. However, due to the complexity of quantifying the aerodynamic interference effects, there is no reliable testing technique so far to incorporate stochastic traffic flow into the wind tunnel tests. Given the existing challenges on the state-of-the-art testing, the present study chooses the vehicle wind coefficients on the ground to demonstrate the methodology. By avoiding bridge-specific complexness of wind coefficients on vehicles, the present study can also provide some more general observations despite the approximation being made. For the vehicle moving in the wind field, the following formulae suggested by Baker (Baker 1987) are used to determine the total wind force and moment coefficients as functions of the yaw angle. 

$C_{D}(\psi)=-a_{3}(1+2 \sin 3 \psi)$
(17a), $\quad C_{S}(\psi)=a_{1} \psi^{0.382}$
$(17 \mathrm{~b}), \quad C_{L}(\psi)=a_{2}(1+\sin 3 \psi)$
$(17 \mathrm{c})$
$C_{R}(\psi)=a_{6} \psi^{0.294}$
$(17 \mathrm{~d}), \quad C_{P}(\psi)=a_{5} \psi^{1.32}$
(17e), $\quad C_{Y}(\psi)=-a_{4} \psi^{1.77}$

in which, the coefficients $a_{1}-a_{6}$ adopt the following values, respectively (Coleman and Baker 1990): $a_{1}=$ 5.2, $a_{2}=0.93, a_{3}=-0.5, a_{4}=2.0, a_{5}=-2.0$ and $a_{6}=7.3$. The sign convention follows the same rule as defined in the original reference.

\subsection{Baseline scenario for vehicle accident analysis}

In this section, the vehicle response will be obtained from bridge-traffic interaction analysis and the representative vehicle for each type will be selected for accident analysis using the total force approach. The steady-state wind speed is $20 \mathrm{~m} / \mathrm{s}$ and moderate traffic flow is moving through the road-bridge-road system. Compared with the high-sided heavy truck and light truck, light sedan car is usually safe from crosswind due to its small frontal area and height. Therefore, only a representative high-sided heavy truck and a light truck are selected for the following vehicle accident investigation. The traveling paths of the two representative vehicles, e.g., the first vehicle of each type in the traffic flow, are presented in Fig. 6.

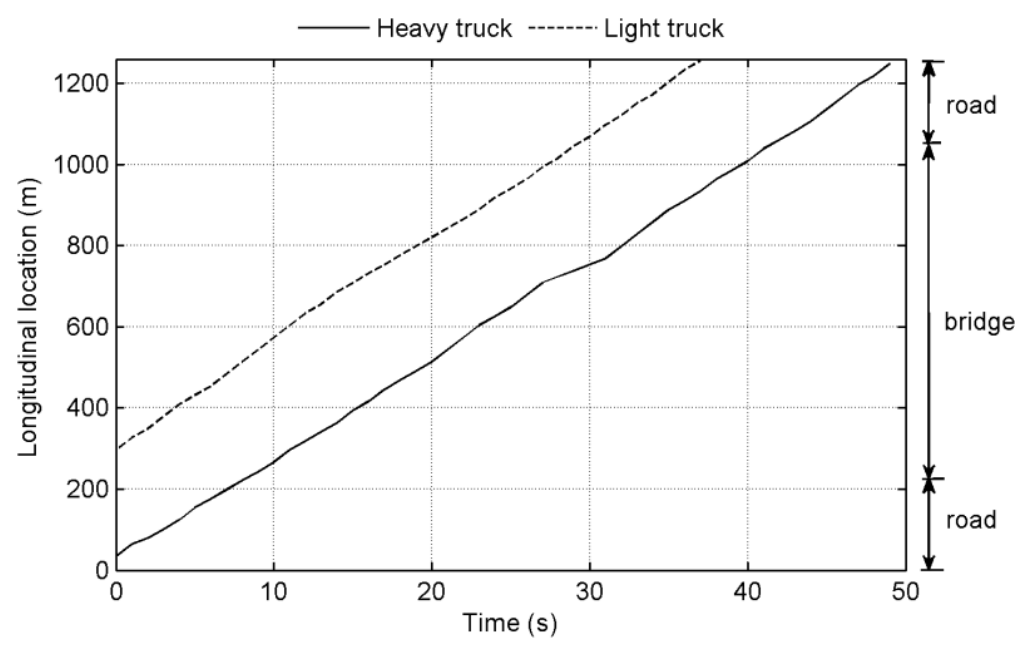

Fig. 6 Instantaneous vehicle location for representative vehicles 
The initial locations for the representative heavy truck and light truck are on the bridge and on the road, respectively. It is seen in Fig. 6 that the driving speeds of vehicles may vary with time following traffic rules. However for traffic flow with a moderate number of vehicles, the vehicles can generally keep a stable driving speed. A horizontal line means that the vehicle is not in the motion. A steeper line section indicates that the vehicle is accelerating while a flatter line section indicates that the vehicle is decelerating from the driving speed in the previous time instant.

The total wind forces acting on the vehicles change with respect to time due to the unsteady characteristics of instantaneous turbulent and vehicle speeds. The four components of total wind force histories during the simulation period on the major $\left(2^{\text {nd }}\right)$ rigid body of the representative heavy truck are given in Fig. 7a-d.

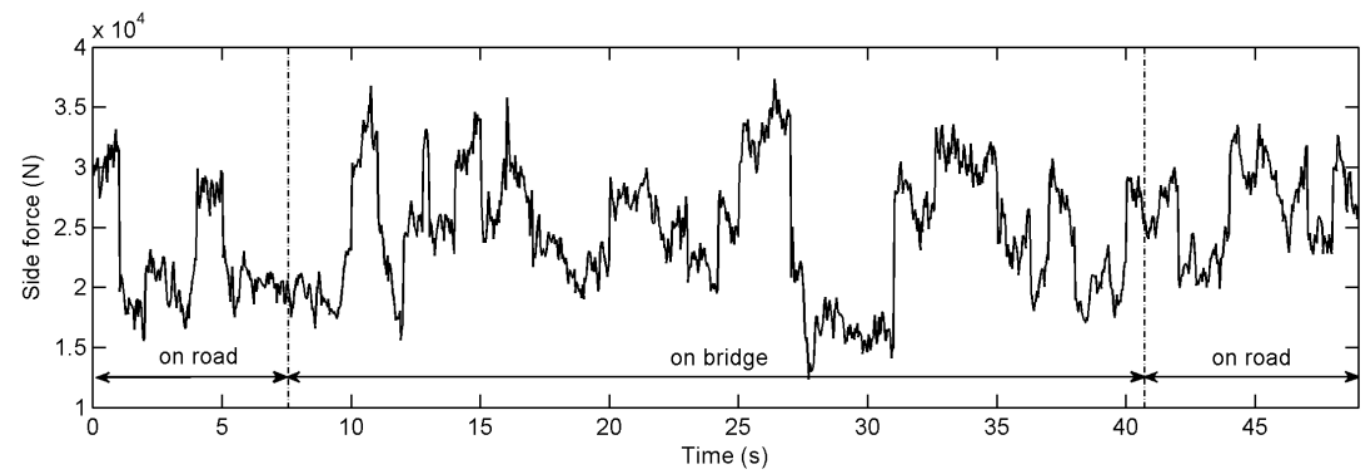

(a) Side force

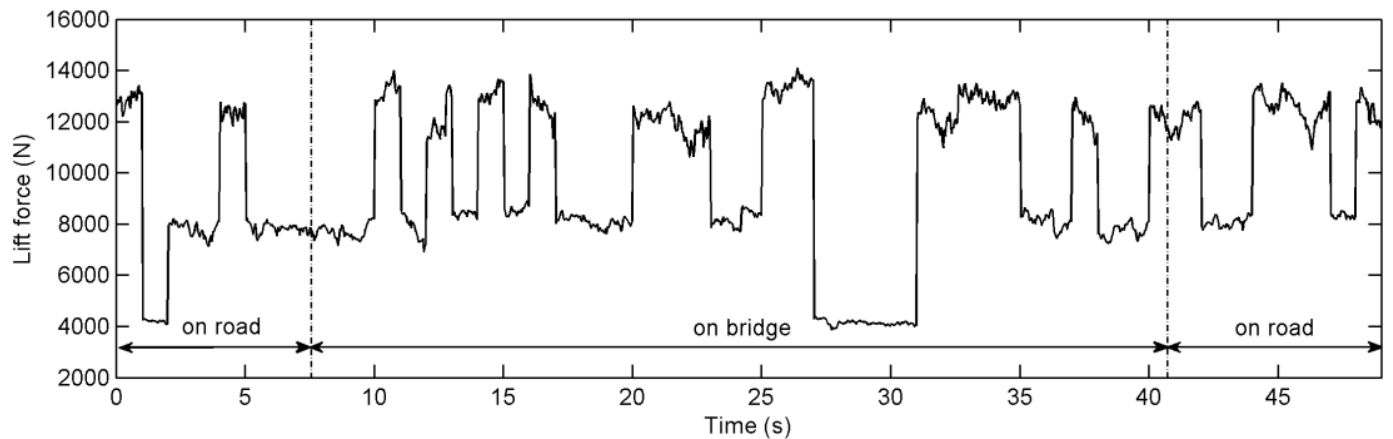

(b) Lift force 


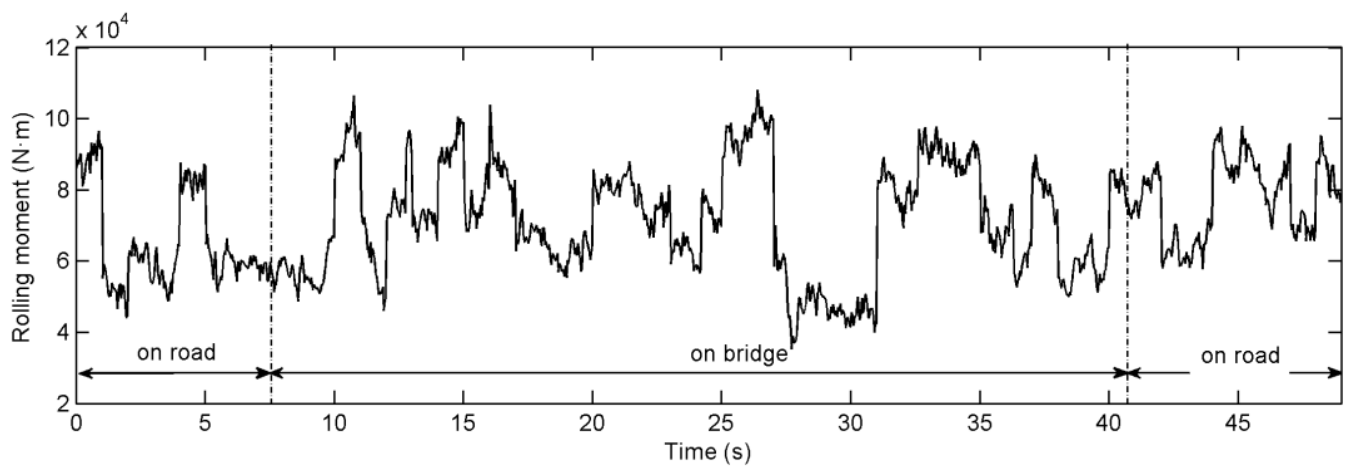

(c) Rolling moment

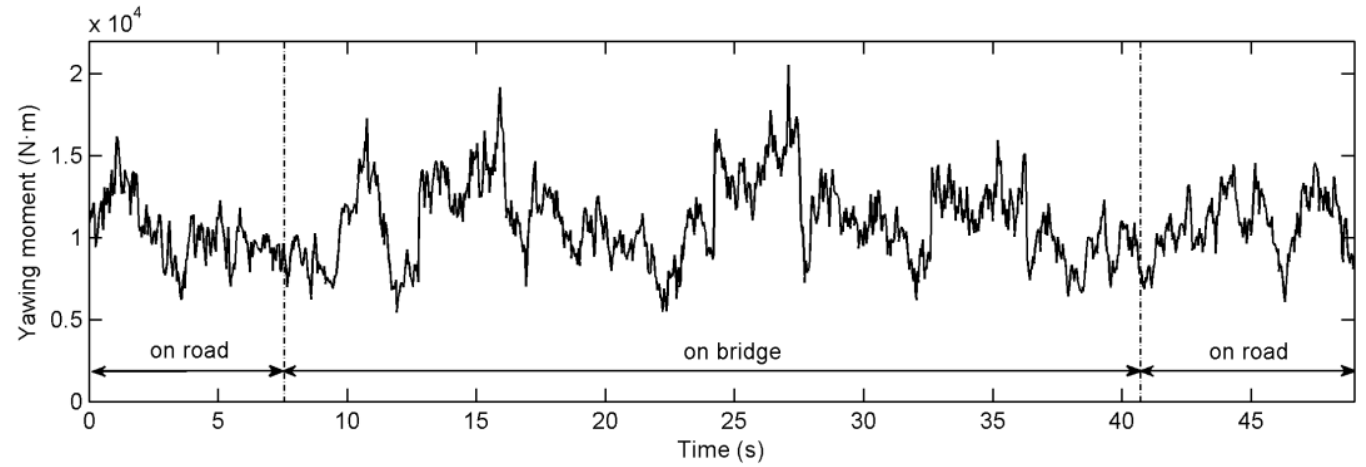

(d) Yawing moment

Fig. 7 Total wind force on the major rigid body of the heavy truck

It is seen in Fig. 7 that the wind forces on the vehicle bodies are non-stationary as they change with respect to both turbulent wind speed and vehicle driving speed. As the vehicle speed changes, the total wind forces acting on the vehicles change significantly. Compared with the other four wind forces, lift and drag forces are more sensitive to the vehicle driving speed. As the vehicle speed drops, both the lift and drag forces drop significantly. When the vehicle speed drops to zero, the cross wind is applied on the vehicle with a yaw angle of $90^{\circ}$ and wind is perpendicular to the longitudinal axis of the driving direction. The time histories of vertical displacement and lateral displacement at the windward side of the $1^{\text {st }}$ wheel set of the heavy truck are demonstrated in Figs. 8a and b, respectively. By Referring to the vehicle 
location in Fig. 6, it is seen that the vehicle enters the bridge at around $7.5 \mathrm{~s}$ and leaves the bridge at around $41.0 \mathrm{~s}$. The vehicle has the largest dynamic response when the vehicle arrives at around the midspan of the bridge. At the start time when wind is firstly applied, the vehicle has reached the local extreme dynamic responses for both vertical and lateral directions. The reaching of local extreme response is due to the sudden presence of wind excitations in various possible scenarios. For instance, when the vehicle passes through the bridge towers, the vehicle will be abruptly shielded from bridge tower and then shortly experience suddenly resumed wind gust. Similar situations may happen to vehicles being passed by other vehicles that are higher and/or larger. As also shown in the following analysis results, the sudden application of wind gust may be critical to the vehicle safety and deserves special attention in order to mitigate the risk of vehicle accidents.

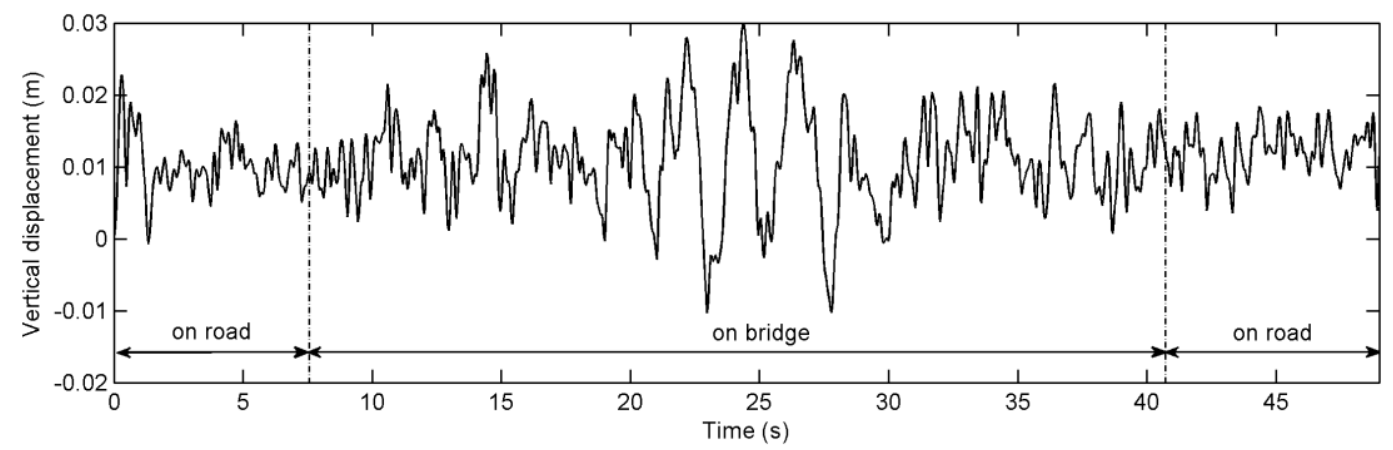

(a) Vertical displacement

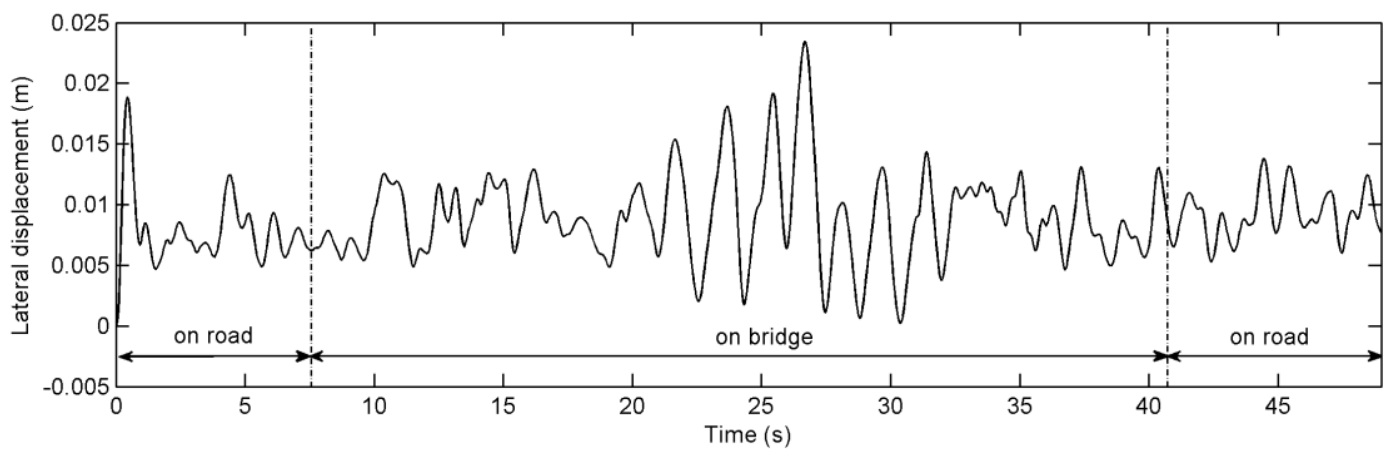

(b) Lateral displacement 
Fig. 8 Dynamic response at the windward side of the $1^{\text {st }}$ wheel set for the heavy truck

\subsubsection{Lift-up accident}

Being lifted up at certain wheel sets is the most common cause of road accident for moving vehicles in windy environment. The vertical contact force time histories at the windward and leeward side of the $3^{\text {rd }}$ wheel set are given for the heavy truck in Figs. 9a and b, respectively.

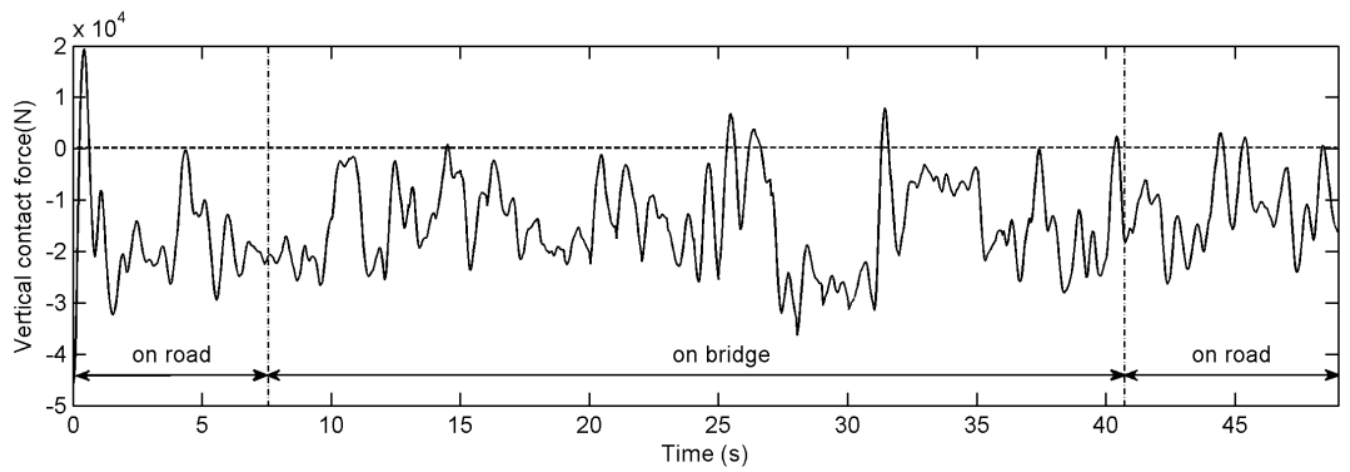

(a) At windward wheel

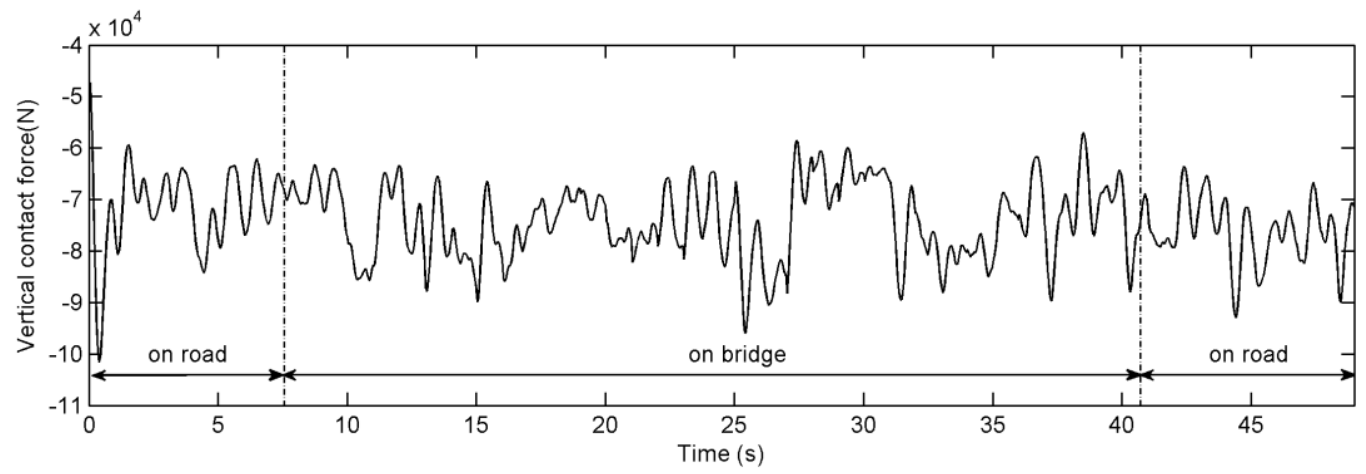

(b) At leeward wheel

Fig. 9 Vertical contact force at windward and leeward side of the $3^{\text {rd }}$ wheel set for the heavy truck

It is seen in Fig. 9 that the side of the wheel in the windward position of the vehicle is more likely to be lifted up in the windy environment. The other side of the vehicle usually remains on the ground unless the vehicle is driven at a very high speed while the wind speed is also in the very high range. Therefore, 
only in very rare cases, both sides of the vehicle wheel set will be lifted up at the same time. The vertical contact forces at windward side of the $1^{\text {st }}$ and $2^{\text {nd }}$ wheel sets are shown in Fig. 10a and b, respectively.

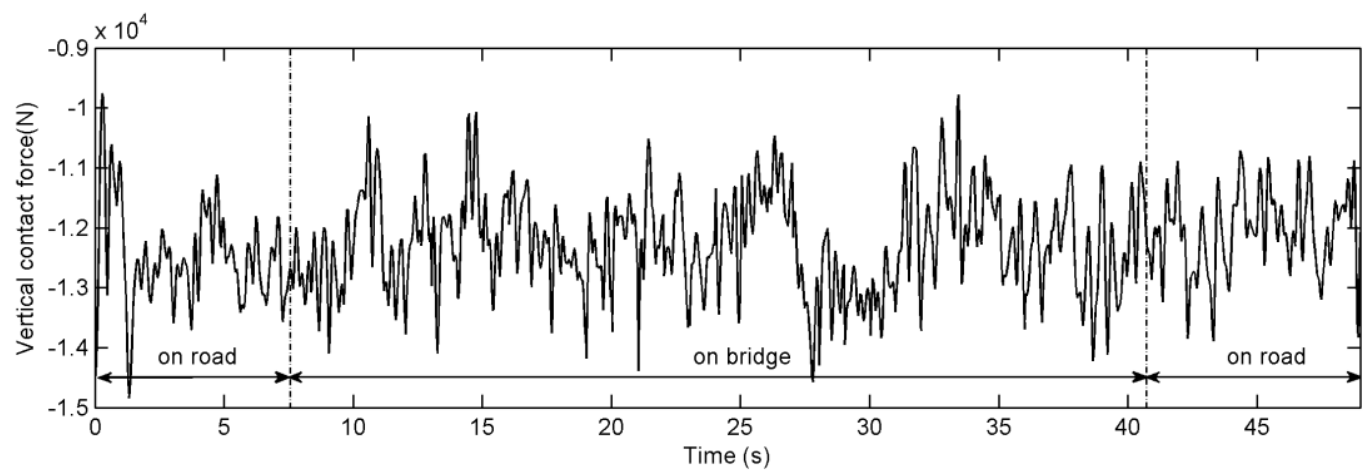

(a) At the windward side of the $1^{\text {st }}$ wheel set

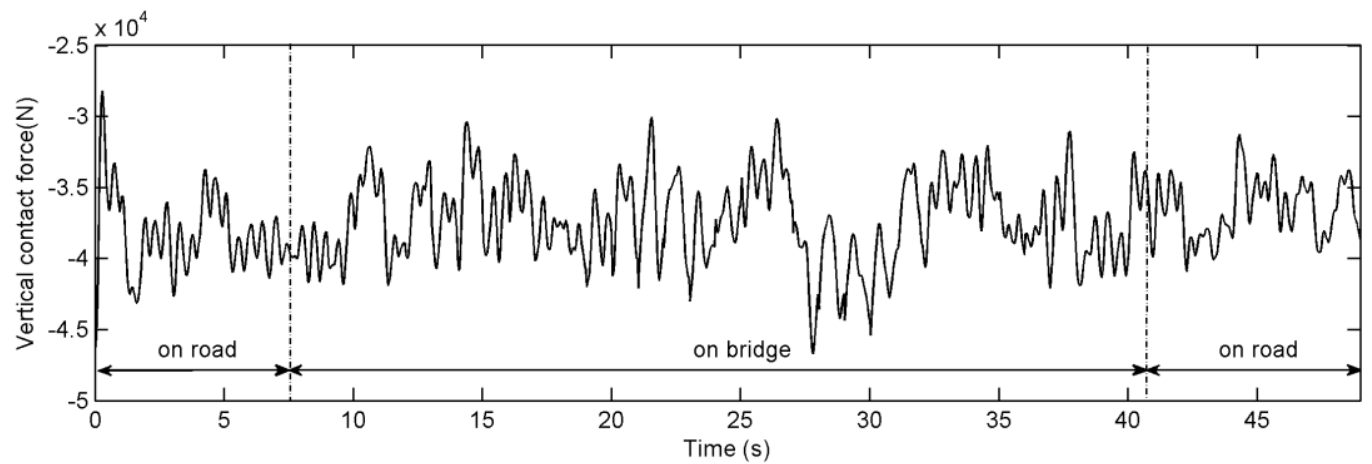

(b) At the windward side of the $2^{\text {nd }}$ wheel set

Fig. 10 Vertical contact force at windward side of the $1^{\text {st }}$ and $2^{\text {nd }}$ wheel set for the heavy truck

It is seen in Fig. 10 that the $1^{\text {st }}$ and $2^{\text {nd }}$ wheel sets at the windward side of the heavy truck remain contact with the ground and will not be lifted up. The results suggest that the windward sides of the $3^{\text {rd }}$ and $2^{\text {nd }}$ wheel sets are the most and least likely ones to be lifted up in the windy environment for highsided heavy truck, respectively. The windward side of the $1^{\text {st }}$ wheel set is less likely to be lifted up as compared to that of the $3^{\text {rd }}$ wheel set while more likely to be lifted up compared with that of the $2^{\text {nd }}$ wheel set. 
Different from the heavy truck with two rigid bodies, the vertical contact forces for the two wheel sets of the light truck are relatively close on the same side (i.e. windward or leeward side), of which the vertical contact force at the $2^{\text {nd }}$ wheel set is a little more likely to be lifted up than that of the $1^{\text {st }}$ wheel set. The vertical contact force histories at the windward side of the $1^{\text {st }}$ and $2^{\text {nd }}$ wheel set for the light truck are shown in Figs. 11 a and b, respectively.

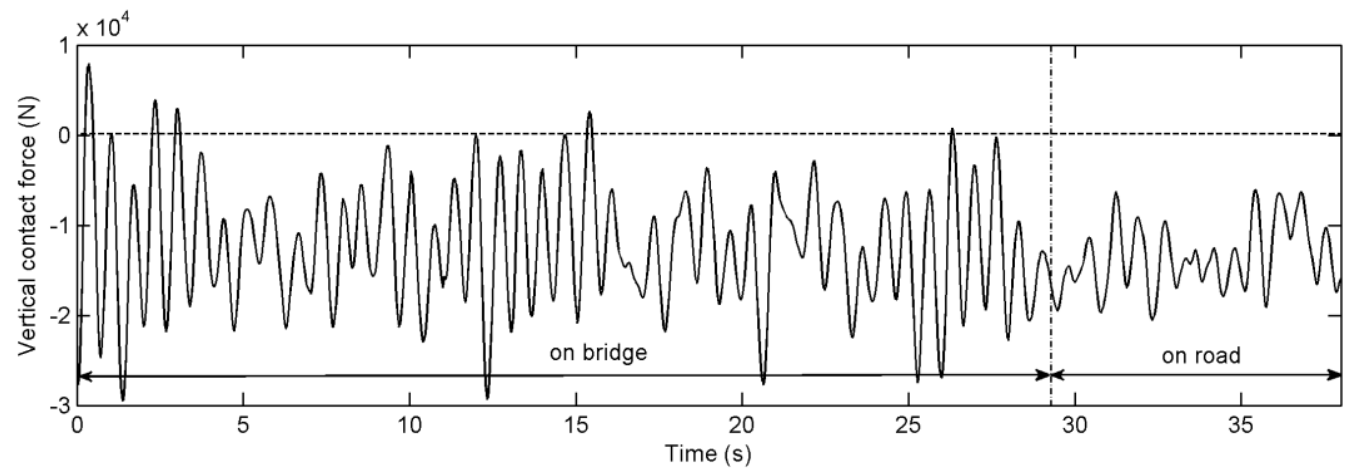

(a) At the $1^{\text {st }}$ wheel set

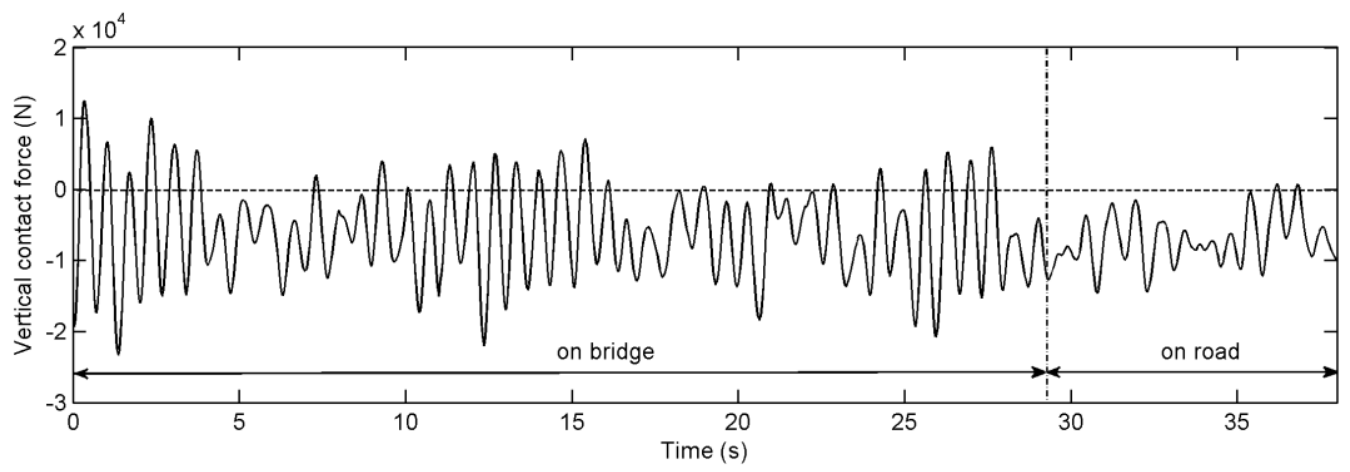

(b) At the $2^{\text {nd }}$ wheel set

Fig. 11 Vertical contact force at windward side of the wheel sets for the light truck

It is found from Figs. 10 and 11 that the lift-up accident can happen to the high-sided heavy truck at the $3^{\text {rd }}$ wheel set and can also happen to the light truck at both wheel sets when the vehicles are driven normally in the windy environment at a steady-state wind speed of $20 \mathrm{~m} / \mathrm{s}$. Comparatively, the light truck is more likely to have lift-up accidents than the heavy truck. It is possibly because of the similar vehicle 
frontal area but much smaller self-weight for the light truck as compared to the heavy truck. As the vehicle speeds up and down, the vertical contact force at the windward side exhibits notable nonstationary characteristics for both the heavy and light trucks. For a certain wheel set, the vertical contact force can be influenced by the vehicle driving speed, turbulent wind speed, relative location on the bridge/road system and the response on the contact point of the bridge. It is shown in Figs. 10 and 11 that the vertical contact force on the ground is more likely to be a positive value at the time instants when the truck is driven on the bridge than on the road. When the vehicle enters the windy environment suddenly $(t$ $=0 \mathrm{~s}$ ), the vertical contact force is very unfavorable and reaches local extreme value in the upward direction. Depending on the specific configuration of the vehicles, it is noted that the patterns of lift-up accidents may differ among vehicles with different wheel set configurations as well as mass distributions.

\subsubsection{Side-slip accident}

When the lateral contact force of a certain wheel exceeds the maximum static friction force, the certain wheel will slip laterally on the bridge or the road. When one side of a certain wheel loses contact with the ground vertically, the side-slip accident will be more likely to happen than in the situation when both sides of the wheel set remain contact with the ground. Unlike the vertical contact force, the leeward and windward sides of the wheel set usually have the similar lateral contact forces at each time instant, which are thus assumed to be the same in the present study. The total lateral contact force is obtained by summing the forces in the lower lateral springs and dampers and the equivalent lateral forces due to yawing moment on the vehicle bodies. The total lateral contact force at the $1^{\text {st }}, 2^{\text {nd }}$ and $3^{\text {rd }}$ wheel set of the heavy truck is demonstrated in Figs. 12a, b and c, respectively. 


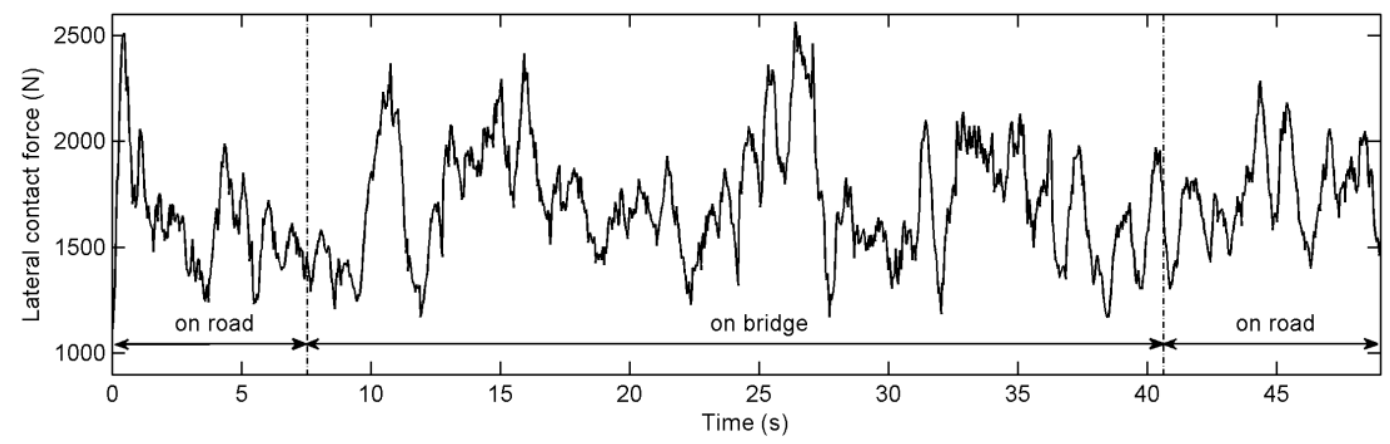

(a) The $1^{\text {st }}$ wheel set

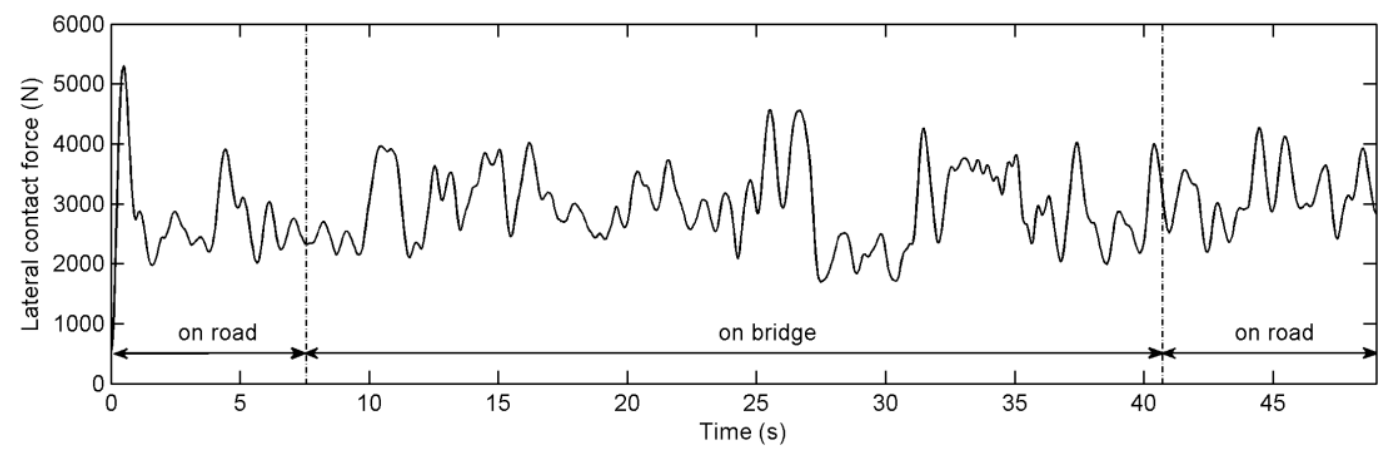

(b) The $2^{\text {nd }}$ wheel set

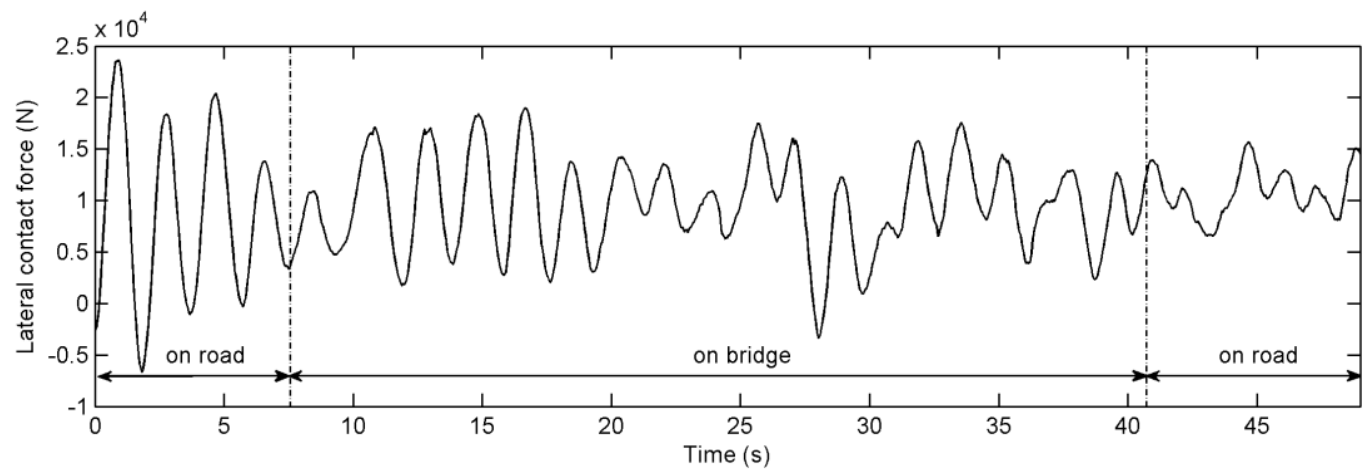

(c) The $3^{\text {rd }}$ wheel set

Fig. 12 The lateral contact force of each wheel set for the heavy truck 
It is seen in Fig. 12 that the global maximum lateral force occurs either at $0 \mathrm{~s}$ when wind forces are applied on the bridge and road suddenly or at around the bridge mid-span. It is found that both the vertical and lateral contact forces have unfavorable values when the vehicle enters into the crosswind environment. When the lateral contact forces at both the windward and leeward wheels cannot resist the maximum static friction force, side-slip accident will occur. The static friction coefficient varies with different types of road conditions. For normal dry road with concrete surface, the static friction coefficient is commonly adopted as 1.0. For wet or snow-covered ground surface, the static friction coefficient may drop to 0.4. For icy ground surface, the static friction coefficient may be less than 0.15 . In this section, the minimum required value of static friction coefficient $\mu_{r i}(\mathrm{t})$ of the $i^{\text {th }}$ wheel set at time $t$ is calculated to resist side-slip at the wheel, which is defined in Eq. (18).

$$
\mu_{r i}(t)=\frac{L_{i L}^{t}(t)+L_{i R}^{t}(t)}{\left|V_{i L}^{t}(t)+V_{i R}^{t}(t)\right|}
$$

In which, the vertical contact force needs to be set to zero if it is a positive value. The obtained static friction coefficients may have positive and negative values at different time instants, which indicate the friction forces are in the positive and negative directions, respectively. The minimum required static friction coefficients for the $1^{\text {st }}, 2^{\text {nd }}$ and $3^{\text {rd }}$ wheel sets of the heavy truck are marked with horizontal lines in Figs. 13a, b and c, respectively.

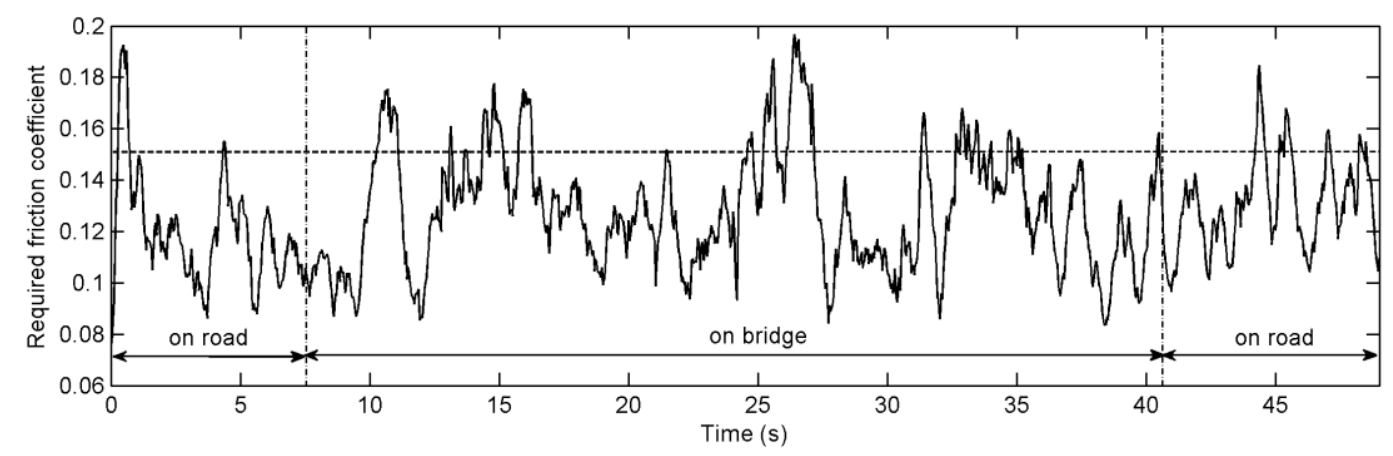

(a) The $1^{\text {st }}$ wheel set 


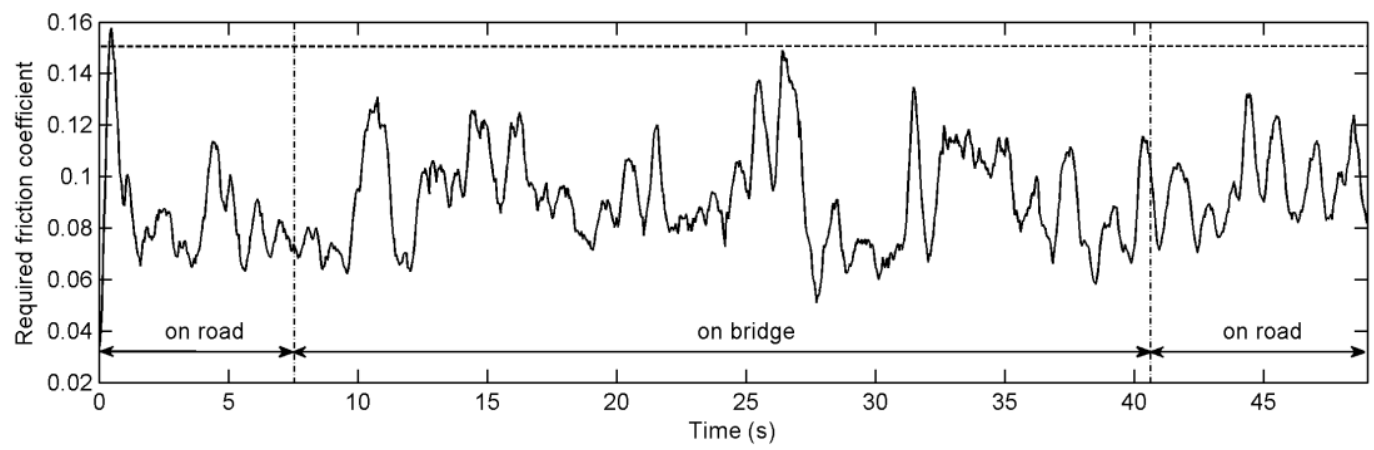

(b) The $2^{\text {nd }}$ wheel set

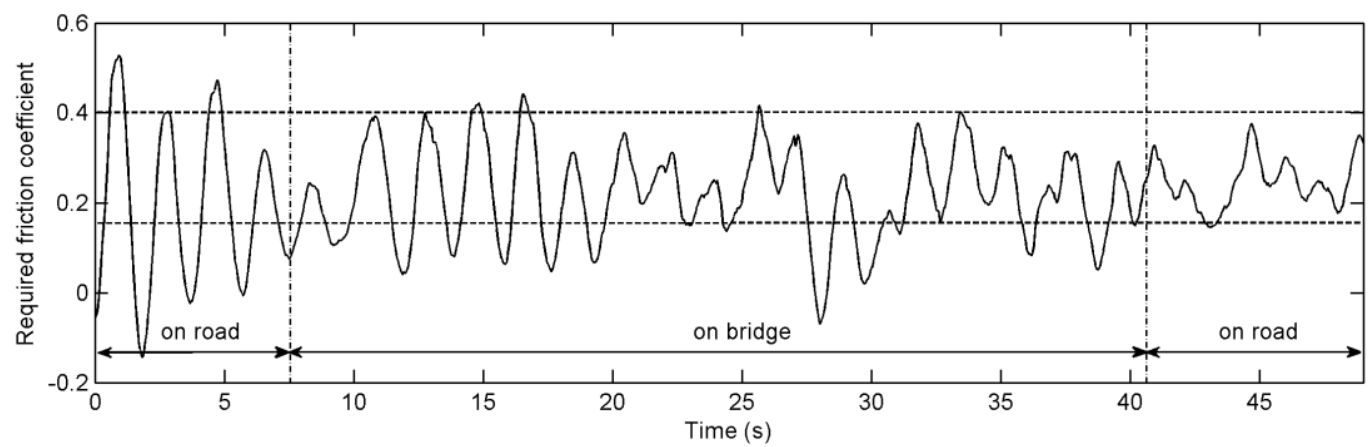

(c) The $3^{\text {rd }}$ wheel set

Fig. 13 Minimum required static friction coefficient for the wheel sets of the heavy truck

It is shown in Fig. 13 that the $3^{\text {rd }}$ wheel set among all the wheels of the heavy truck is most likely to experience side-slip during the analysis. For the $3^{\text {rd }}$ wheel set, the vehicle is safe without slipping laterally on the dry ground surface with a static friction coefficient around 1.0. If the vehicle is driven on slippery road surface covered with water or snow, the static friction coefficient may drop below 0.4 and the $3^{\text {rd }}$ wheel set will have side-slip accidents from time to time. On the road surface with a static friction coefficient less than 0.15 , the $3^{\text {rd }}$ wheel set will have lateral slipping frequently. The minimum required static friction coefficients for the $1^{\text {st }}$ and $2^{\text {nd }}$ wheel sets are very low, and therefore normally no side-slip accident will occur to the two wheel sets unless the friction coefficient on the ground surface drops below 
0.2. Compared with the $2^{\text {nd }}$ wheel set, the $1^{\text {st }}$ wheel set will experience side-slip frequently on the icy road surface with a static friction coefficient less than 0.15 .

The time histories of the minimum required static friction coefficient at the $1^{\text {st }}$ and $2^{\text {nd }}$ wheel set for the light truck are shown in Fig. 14a and b, respectively. It can be seen that the light truck will not experience side-slip accidents on dry road surface for both wheel sets. The $1^{\text {st }}$ wheel set is slightly more disadvantageous for side-slip accidents than the $2^{\text {nd }}$ one. On slippery road covered by rain flow or snow with a static friction coefficient less than 0.4 , both wheel sets may experience side-slip at certain time instants. On icy road with a static friction coefficient less than 0.15 , the side-slip at both wheels will occur frequently. Therefore, it is apparent that the side-slip accident is influenced by the lateral contact force, vertical contact force and ground friction condition. The lateral and vertical contact forces are closely related to the bridge-vehicle coupling interactions when the vehicle is driven on the bridge. By referring to the vertical contact force histories in Fig. 11, it is found that side-slip accidents may or may not be associated with lift-up accidents at the same wheel set.

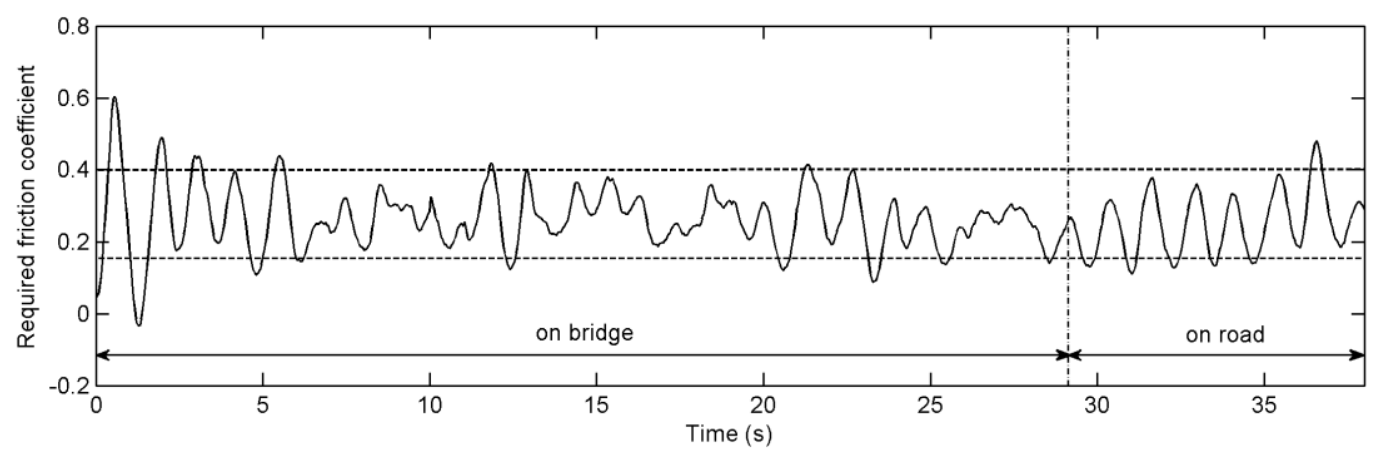

(a) The $1^{\text {st }}$ wheel set 


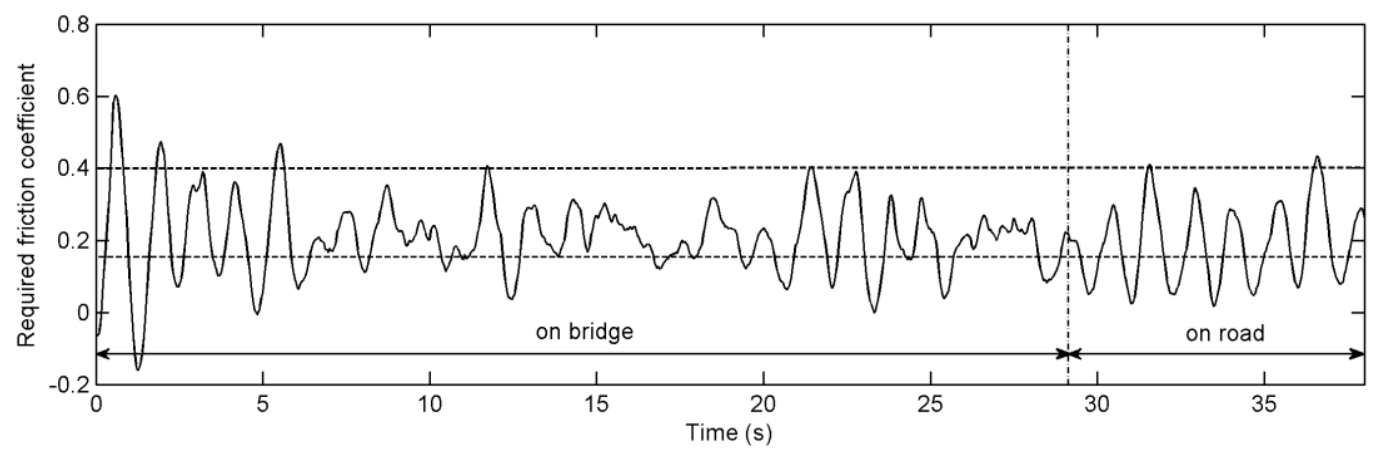

(b) The $2^{\text {nd }}$ wheel set

Fig. 14 Minimum required static friction coefficient for the wheel sets of the light truck

\subsubsection{Yawing accident}

Yawing accident is defined as the rotational movement of the vehicle around the vertical axis. For the heavy truck, the $1^{\text {st }}$ and $2^{\text {nd }}$ wheel sets will not slip laterally while the $3^{\text {rd }}$ wheel set may experience sideslip at certain time instants if the friction condition on road surface is between 0.20 and 0.50 . In this situation, the vehicle body will have a rotation around the vertical axis of the pivot connecting the two rigid bodies. If the static friction coefficient is around 0.15 , the $1^{\text {st }}$ and $3^{\text {rd }}$ wheel sets will have side-slip at certain time instants while the $2^{\text {nd }}$ wheel set remains safe laterally. The yawing accident may be considered to happen around the vertical axis at the $2^{\text {nd }}$ wheel set for the first rigid body and at the pivot for the second rigid body.

For the light truck that consists of only one rigid body, yawing accidents may happen around the vertical axis at the $2^{\text {nd }}$ wheel set considering the fact that the $2^{\text {nd }}$ wheel set stands still while the $1^{\text {st }}$ wheel set experiences side-slip at certain time instants on slippery road surface, e.g., at around $3.0 \mathrm{~s}$. At the time instants when both wheel sets slip laterally on certain road surface conditions, the yawing accident may not occur considering that the side-slip of the wheel happens in the same direction. It is indicated that yawing accident is closely related to the side-slip accident at the wheel sets. Since the side-slip accidents of different wheel sets usually happen in the same direction, yawing accident is typically represented by 
the situation when at least one wheel set remains still laterally on the ground while other wheel sets slip laterally.

\subsection{Influence of the presence of multiple vehicles}

As discussed earlier, existing studies usually investigated the situation when a single vehicle is driven through a bridge at a constant speed. When more realistic traffic scenario is simulated, the presence of multiple vehicles on the bridge at a time becomes very likely. It is thus becomes interesting to evaluate the effects of adopting more realistic multi-vehicle scenarios on traffic safety versus that under singlevehicle assumption. To achieve this, this study firstly conducted two comparative case studies without considering wind excitations: the case of traffic flow with multiple vehicles and the case considering only one single vehicle. In the traffic flow case, the simulated moderate traffic flow passes the road-bridgeroad system and the response of the representative heavy truck is investigated. In the single-vehicle case, the same heavy truck is assumed to move through the road-bridge-road system with exactly the same instantaneous driving speed at any time instant as those in the traffic flow case of the stochastic traffic flow. Particularly, the time histories of the vertical and lateral contact forces at the $3^{\text {rd }}$ wheel of the representative heavy truck are shown in Figs. 15a and b, respectively.

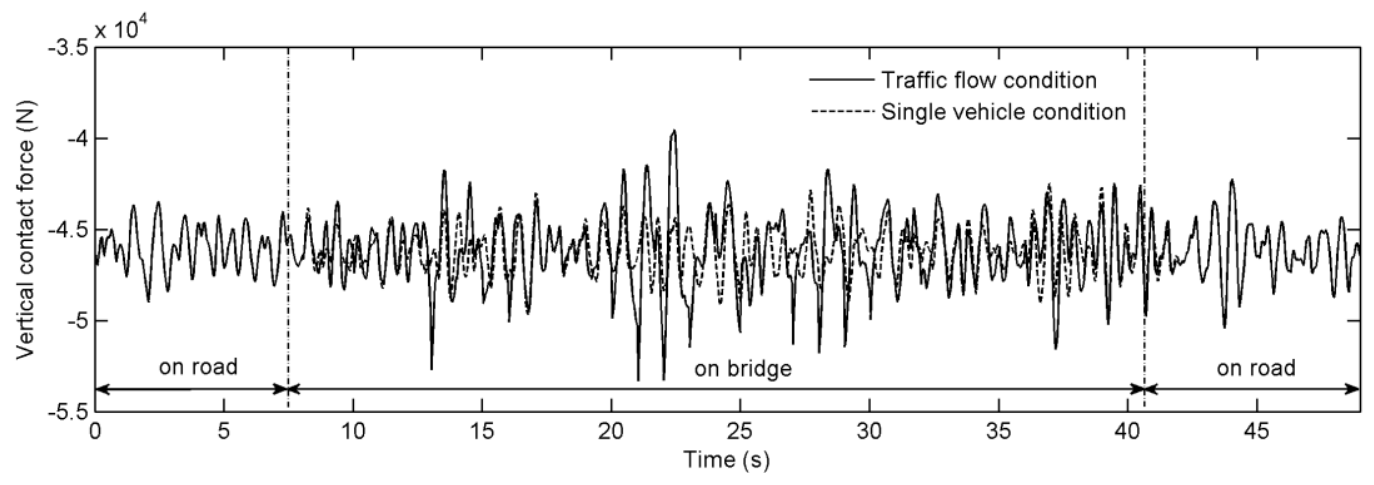

(a) Vertical contact force 


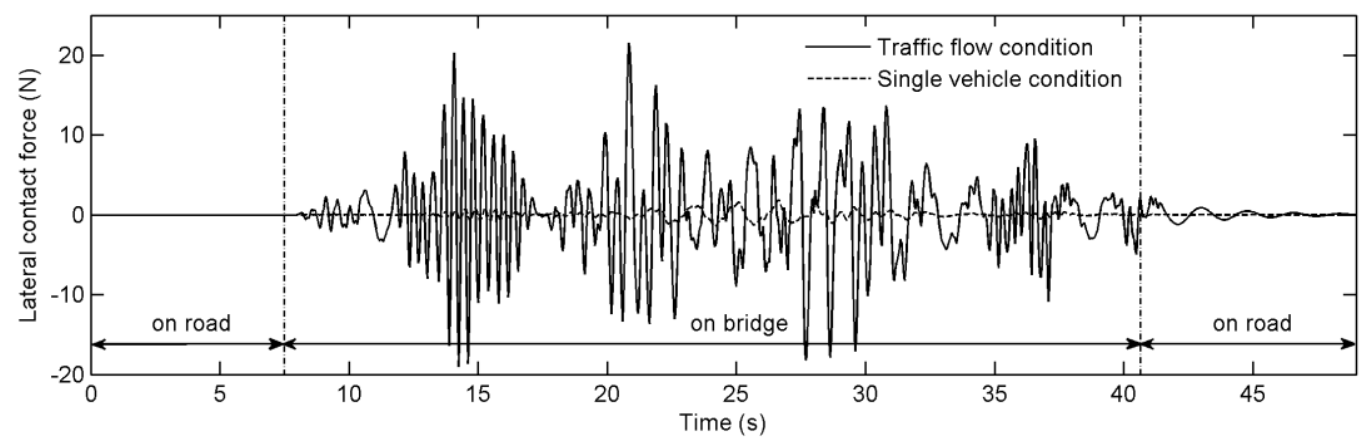

(b) Lateral contact force

Fig. 15 Vertical and lateral contact force for the two comparative cases (No wind)

It is found in Fig. 15a that the vertical contact forces of the vehicle are exactly the same for the two comparative cases when the vehicle is driven on the first approaching road section before entering the bridge. The differences of the vertical contact force between the two cases gradually disappear after the vehicle leaves the bridge and moves on the second approaching road section. When wind excitations are not applied, the vertical contact forces in the single-vehicle case do not have significant variation between the scenarios when the vehicle is driven on the bridge or on the road. However in the traffic flow case, the variation in the vertical contact force of the heavy truck is much larger when the vehicle moves on the bridge than on road, especially when the vehicle moves at around bridge mid-span.

As shown in Fig. 15b, the lateral contact forces of the vehicle in both cases are zero when the vehicle is driven on the first approaching road section before entering the bridge. After the vehicle enters the bridge, the lateral contact force starts to oscillate around its mean value and the variation amplitude gradually increases as the vehicle moves towards the mid-span of the bridge in the single-vehicle case. Such a phenomenon is observed differently for the traffic flow case, likely due to the fact that the lateral contact force of the vehicle being studied is considerably influenced by the vibration of other vehicles. In both cases, the lateral contact forces gradually damp out when the vehicle leaves the bridge and moves on the second approaching road section. When the vehicle is driven on the bridge, the lateral contact forces 
in the traffic flow case are significantly larger than those in the single-vehicle case. It is indicated that the presence of other multiple vehicles may increase the dynamic response of the vehicle in investigation in both vertical and lateral directions.

As a second step, two similar comparative cases are investigated when wind excitations are applied with a steady-state wind speed of $20 \mathrm{~m} / \mathrm{s}$. Figs. 16a and b demonstrate the comparative time histories of the vertical and lateral contact forces at the $3^{\text {rd }}$ wheel of the heavy truck, respectively.

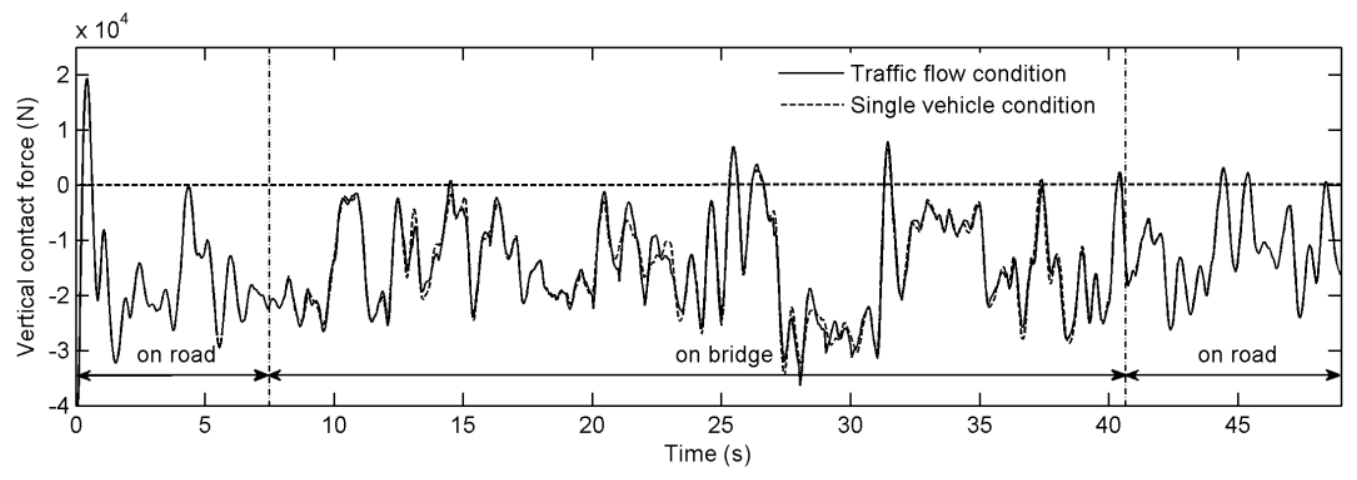

(a) Vertical contact force

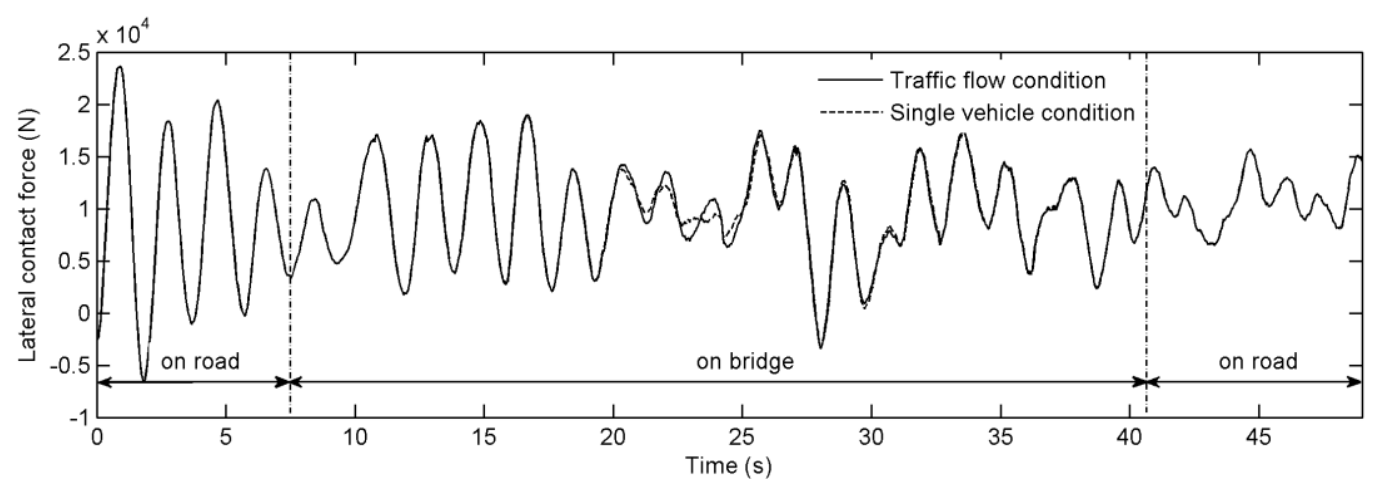

(b) Lateral contact force

Fig. 16 Vertical and lateral contact force for the two comparative cases (Mean wind speed = $20 \mathrm{~m} / \mathrm{s}$ )

By comparing Figs. 15 and 16, it is seen that wind excitations at a steady-state wind speed of $20 \mathrm{~m} / \mathrm{s}$ contributes to a large portion of the total vertical and lateral contact forces on the vehicle. The relative 
differences between the two comparative cases for vertical and lateral contact forces are generally smaller than those between the cases without wind excitations. This is partly due to the different characteristics of dynamic oscillation of the bridge and vehicles under windy and no-wind scenarios. It is also partly due to the fact that the wind force acting on vehicles, especially lift force, is much more dependent on the instantaneous vehicle driving speed rather than the movement of the supporting structure, as discussed earlier. It is demonstrated in Figs. 16a and b that the vertical and lateral contact forces of the vehicle in the traffic flow case are larger than those in the single-vehicle case at most time instants when the vehicle is driven on the bridge. For instance, at around $14.1 \mathrm{~s}$, the vertical contact forces of the vehicle in the traffic flow case and single-vehicle case are $851 \mathrm{~N}$ and $-1692 \mathrm{~N}$, respectively. The lift-up at the windward side of the $3^{\text {rd }}$ wheel may happen when multiple vehicles are present whereas it may not happen when only the single vehicle is involved. However, due to the complex interaction in the bridge-traffic-wind system, there are a few exceptions at which the contact forces of the vehicle are more disadvantageous in the single-vehicle case than those in the traffic flow case, especially for the vertical contact force. It becomes apparent that the presence of multiple vehicles on the bridge can significantly influence the vehicle accident condition on the bridge in the windy environment, underscoring the necessity of carrying out the study based on more realistic traffic simulation.

\subsection{Influence of the dynamic initial states of vehicle when wind excitations are applied}

It is found that a high-sided vehicle may be lifted up or slip laterally when the vehicle enters the crosswind gust suddenly. Previous results assume that the vehicle enters the wind environment at the starting point of the analysis. However in reality, the wind gust may occur at any time during the process when vehicles pass through the bridge. To investigate the influence of the initial states when wind excitations are applied, sudden wind gust is assumed to occur at time instants $22.04 \mathrm{~s}$ and $22.44 \mathrm{~s}$, at which the vertical contact force at the windward side of the $3^{\text {rd }}$ wheel of the heavy truck reaches the lowest and highest values when the vehicle is driven around the mid-span of the bridge, respectively, as shown in Fig. 15a. The vertical contact force time histories at the windward side of the $3^{\text {rd }}$ wheel for the 
heavy truck are demonstrated in Fig. 17 for the cases with the two initial states as the start of wind excitations.

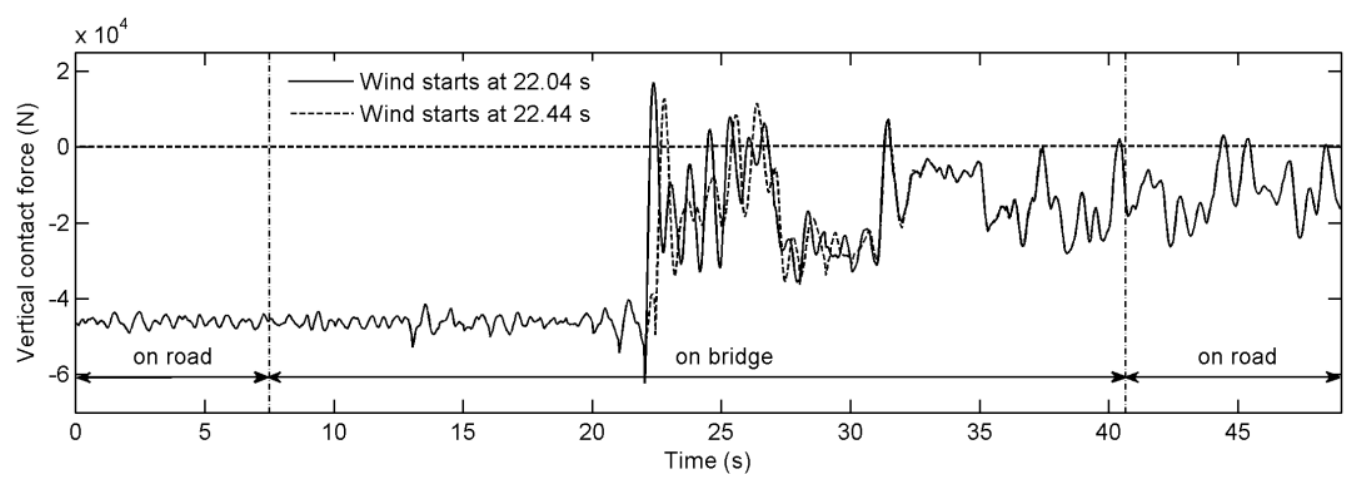

Fig. 17 Vertical contact force with different start time of wind excitations

In Fig. 17, the vertical contact forces at around $22.5 \mathrm{~s}$ when the wind starts at $22.04 \mathrm{~s}$ and $22.44 \mathrm{~s}$ are $17,040 \mathrm{~N}$ and $12770 \mathrm{~N}$, respectively. The results show that the vertical contact force at around the wind start time will be more disadvantageous if wind is applied suddenly at the time when the vehicle wheel reaches the lowest position relative to the bridge and starts to move upward. If the vehicle wheel is at the upward position and starts to move downward when wind is applied abruptly, the increase of the vertical contact force in the upward direction will be less significant. During the following several seconds, the vehicle vibration becomes steady and the difference between the two cases becomes smaller. After around $35 \mathrm{~s}$, there is hardly any difference for the response in two cases and the influence due to the different start time of wind excitations gradually disappear.

\section{Conclusions}

An integrated dynamic interaction and accident assessment framework was proposed based on the developed fully coupled bridge-traffic system formulation. Different from existing studies on vehicle accident analysis, the present study considered realistic stochastic traffic flow passing through a long-span bridge, in which multiple vehicles are on the bridge at the same time and the vehicles can accelerate and 
decelerate following certain traffic rules. The traffic accident analysis of any vehicle on a long-span bridge was conducted based on the fully coupled bridge-traffic interaction analysis of all vehicles of the stochastic traffic on the bridge simultaneously, providing more realistic insights than existing studies. The proposed approach is able to identify the vehicle lift-up, side-slip and yawing accidents within the same integrated simulation framework for the dynamic interaction analysis between the bridge and vehicles. Taking a prototype long-span cable-stayed bridge and three types of vehicles as example, this study evaluated the safety condition corresponding to each type of accidents for the representative heavy truck and light truck. The case study demonstrates that the proposed approach can be used to efficiently assess the safety of any vehicle of the traffic flow on the long-span cable-stayed bridge subjected to crosswind. Finally the influences from the multi-vehicle presence and dynamic initial states of wind excitations on traffic safety were numerically studied. Some specific observations are summarized in the following.

- The contact force of wheels is influenced by vehicle driving speed, turbulent wind speed, location on the bridge/road and the response on the contact point of bridge.

- The vehicle is more likely to be lifted up at the start time of wind excitations than any other time instants when other conditions remain the same.

- The vehicle is more likely to be lifted up or slip laterally when the vehicle is driven on a longspan bridge than on a road with other conditions remaining the same.

$\circ$ The $3^{\text {rd }}$ wheel set of the heavy truck is the most disadvantageous one in terms of lift-up or sideslip accidents. The $2^{\text {nd }}$ wheel set is more disadvantageous to be lifted up than the $1^{\text {st }}$ wheel set for the light truck. The side-slip risks of the two wheel sets for the light truck are similar.

- Side-slip accidents will not be likely to happen on dry road surface for both the heavy truck and light truck when the steady-state wind speed is no more than $20 \mathrm{~m} / \mathrm{s}$, while it may happen on slippery road surface, such as being covered with snow, water or ice. 
○ Yawing accident is usually associated with the side-slip of the wheels. Depending on the specific position where the lateral slipping at the wheels may occur, yawing accidents can happen in several possible manners for both the heavy and light trucks.

$\circ$ The presence of multiple vehicles usually increases the variation of the dynamic response of the vehicle of interest in both vertical and lateral directions. The presence of multiple vehicles on the bridge may significantly influence the accident risks of the vehicles on the bridge in the windy environment.

- The vehicle accident condition on the bridge in the windy environment is closely related to the dynamic initial states of the vehicle when sudden wind excitations are applied.

The present study has made important efforts on exploring traffic safety by considering the complex dynamic interactions of the bridge and stochastic traffic system. However, due to the remaining technical challenges on obtaining realistic wind parameters from experiments, the aerodynamic interference effects on the wind coefficients of the bridge and vehicles in the traffic flow were not considered. Besides, the wind speed shielding effects on the vehicles due to the presence of bridge towers and the other vehicles passing by were neglected. To obtain accurate wind coefficients of bridge and moving stochastic traffic system still remains as a challenge to the wind engineering community. It is the future task that the aerodynamic interference and shielding effects due to stochastic traffic flow can be incorporated to obtain more accurate results.

\section{Acknowledgement}

This material is based upon work supported by the National Science Foundation under Grant No. CMMI1335571. Any opinions, findings, and conclusions or recommendations expressed in this material are those of the investigators and do not necessarily reflect the views of the National Science Foundation. 


\section{References}

Baker, C. J. (1986). "Simplified analysis of various types of wind induced road vehicle accidents." $J$. Wind Eng. Ind. Aerodyn., 22 (1), 69-85.

Baker, C. J. (1987). "Measures to control vehicle movement at exposed sites during windy periods." J. Wind Eng. Ind. Aerodyn., 25 (2), 151-161.

Baker, C. J. (1991). "Ground vehicles in high cross winds. 3: The interaction of aerodynamic forces and the vehicle system.” J. Fluids Struct., 5, 221-241.

Baker, C. J. (1994). "Quantification of accident risk for road vehicles in cross winds." J. Wind Eng. Ind. Aerodyn., 52(1-3), 93-107.

Cai, C. S., and Chen, S. R. (2004). "Framework of vehicle-bridge-wind dynamic analysis." J. Wind Eng. Ind. Aerodyn., 92 (7-8), 579-607.

Chen, F. and Chen, S. R. (2010). "Simulation-based assessment of vehicle safety behavior under hazardous driving conditions.” J. Transportation Eng., ASCE, 136 (4), 304-315.

Chen, S. R., and Cai, C. S. (2004). “Accident assessment of vehicles on long-span bridges in windy environments.” J. Wind Eng. Ind. Aerodyn., 92 (12), 991-1024.

Chen, S. R., Cai, C. S., and Wolshon, B. (2009). "From normal operation to evacuation: Single-vehicle safety under adverse weather, topographic and operational conditions." Nat. Hazards Rev.,10 (2), 68-76.

Chen, S. R. and Wu, J. (2010). "Dynamic performance simulation of long-span bridge under combined loads of stochastic traffic and wind", J. Bridge Eng., ASCE, 15(3), 219-230.

Chen, S. R. and Wu, J. (2011). "Modeling stochastic live load for long-span bridge based on microscopic traffic flow simulation”, Computer \& Structures, 89, 813-824. 
Coleman, S. A., and Baker, C. J. (1990). "High sided road vehicles in cross wind." J. Wind Eng. Ind. Aerodyn., 36 (1990), 1383-1392.

Guo, W. H., and Xu, Y. L. (2006). "Safety analysis of moving road vehicles on a long bridge under crosswind.”J. Eng. Mech., 132 (4), 438-446.

Han, Y., Cai, C.S., et al. (2014). "Effects of aerodynamic parameters on the dynamic responses of road vehicles and bridges under cross winds ”, J. Wind Eng. Ind. Eng. Ind. Aerodyn. 134 (2014), 78-95.

Huang, D. Z. and Wang, T. L. (1992). "Impact analysis of cable-stayed bridges", Computers and Structures, 31, 175-183.

Lin, Y. K. and Yang, J. N. (1983). "Multimode bridge response to wind excitations", J. Eng. Mech., ASCE, 109 (2), 586-603.

USDOT. (2005). "Report to Congress on the large truck crash causation study." Federal Motor Carrier Safety Administration, U.S. Dept. of Transportation, Washington, D.C.

Zhou, Y. F., and Chen, S. R. (2014a). "Dynamic simulation of long-span bridge-traffic system subjected to combined service and extreme loads". J. Struct. Eng., ASCE, 10.1061/(ASCE)ST.1943-541X.0001188, 04014215.

Zhou, Y. F., and Chen, S. R. (2014b). "Framework of fully-coupled nonlinear dynamic simulation of long-span cable-stayed bridges subjected to cable-loss incidents”. J. Struct. Eng., ASCE, under review. 